\title{
OPEN In vivo melanin 3D quantification and z-epidermal distribution by multiphoton FLIM, phasor and Pseudo-FLIM analyses
}

\author{
Ana-Maria Pena ${ }^{1} \bowtie$, Etienne Decencière ${ }^{2}$, Sébastien Brizion ${ }^{1}$, Peggy Sextius ${ }^{1}$, \\ Serge Koudoro ${ }^{2}$, Thérèse Baldeweck ${ }^{1}$ \& Emmanuelle Tancrède-Bohin ${ }^{3,4}$
}

Characterizing melanins in situ and determining their 3D z-epidermal distribution is paramount for understanding physiological/pathological processes of melanin neosynthesis, transfer, degradation or modulation with external UV exposure or cosmetic/pharmaceutical products. Multiphoton fluorescence intensity- and lifetime-based approaches have been shown to afford melanin detection, but how can one quantify melanin in vivo in 3D from multiphoton fluorescence lifetime (FLIM) data, especially since FLIM imaging requires long image acquisition times not compatible with 3D imaging in a clinical setup? We propose an approach combining (i) multiphoton FLIM, (ii) fast image acquisition times, and (iii) a melanin detection method called Pseudo-FLIM, based on slope analysis of autofluorescence intensity decays from temporally binned data. We compare Pseudo-FLIM to FLIM bi-exponential and phasor analyses of synthetic melanin, melanocytes/keratinocytes coculture and in vivo human skin. Using parameters of global 3D epidermal melanin density and z-epidermal distribution profile, we provide first insights into the in vivo knowledge of 3D melanin modulations with constitutive pigmentation versus ethnicity, with seasonality over 1 year and with topical application of retinoic acid or retinol on human skin. Applications of Pseudo-FLIM based melanin detection encompass physiological, pathological, or environmental factors-induced pigmentation modulations up to whitening, anti-photoaging, or photoprotection products evaluation.

Characterizing melanins in their native environment and determining their $3 \mathrm{D}$ distribution in epidermis is paramount for understanding physiological processes of melanin neosynthesis, transfer and degradation and their modulation with external factors such as UV light exposure, topical products application, or skin diseases.

Human skin melanins, are a mixture of eumelanins and pheomelanins ${ }^{1-5}$ produced in melanosomes organelles within the melanocytes ${ }^{6}$. Stage IV melanized melanosomes are transferred to keratinocytes and their number, size and distribution within the epidermis contribute along with other chromophores mainly hemoglobin to human skin color ${ }^{1}$.

Currently, the gold standard method for melanin quantification in skin is high-performance liquid chromatography (HPLC) chemical analysis of melanin degradation products ${ }^{7,8}$. Although very specific, it requires $e x$ vivo samples degradation and provides no information on melanin's epidermal distribution. Fontana-Masson staining of transverse skin sections allows insights in 2D melanin distribution and content (e.g. ${ }^{9}{ }^{10}$ ) but has the drawback of non-specific stratum corneum (SC) staining, while Warthin-Starry stain may provide a more sensitive and specific melanin detection ${ }^{11}$. Transmission electron microscopy enables melanosomes pattern analysis within epidermal cells and its variations according to skin phenotype (e.g. $\left.{ }^{10}\right)$.

Assessing melanin content and distribution in vivo, in 3D, is still a challenging topic. Over the years, the possibility to provide a non-invasive melanin detection based on its optical properties, broad-band UV and visible absorption $^{12,13}$, fluorescence emission spectrum and lifetime ${ }^{14,15}$, was investigated using different techniques. In confocal reflectance microscopy ${ }^{16}$, melanin contrast arises from refractive index changes between melanin pigment and other constituents ${ }^{17-19}$, but cellular membranes and corneocytes also exhibit similar reflection

${ }^{1} L^{\prime}$ Oréal Research and Innovation, 1 Avenue Eugène Schueller, BP22, 93601 Aulnay-sous-Bois, France. ${ }^{2}$ MINES ParisTech - PSL Research University, Fontainebleau, France. ${ }^{3}$ L'Oréal Research and Innovation, Campus Charles Zviak RIO, 9 rue Pierre Dreyfus, Clichy, France. ${ }^{4}$ Service de Dermatologie, Hôpital Saint-Louis, Paris, France. ${ }^{\boxplus}$ email: ana-maria.pena@rd.loreal.com 
signals, making specific detection and quantification impossible. Studies using spontaneous Raman spectroscopy found eumelanin and pheomelanin to have specific peaks and the $2000 \mathrm{~cm}^{-1}$ peak of pheomelanin located within the "silent region" of the Raman spectrum seems to offer a straightforward route to specific non-invasive 3D pheomelanin detection in skin samples by CARS (Coherent anti-Stokes Raman Scattering) imaging ${ }^{20}$. The Raman bands $550-1200$ and $1650-2300 \mathrm{~cm}^{-1}$ were also shown to be significant to predict the ratio of eumelanin subunits ${ }^{21}$. Recently, it was proposed that a combined analysis of specific Raman bands along with the NIR one-photon excited skin autofluorescence ${ }^{22,23}$ could be used to estimate an xz depth-profile of melanin fraction in vivo ${ }^{24}$ but this method lacks spatial localization of melanin within the cells and cannot image the entire epidermis. Pump-probe imaging has also been proven of value in analyzing melanin within $2 \mathrm{D}$ thin skin sections of pigmented lesions, namely for its eumelanin/pheomelanin discrimination ${ }^{25-27}$. As the dominant absorber within epidermis, melanin also provides a strong third harmonic generation (THG) signal in the basal layers and, using an in vivo calibration method, a melanin mass density can be estimated from THG images ${ }^{28}$. However, it is not clear how melanin THG signal is discriminated from the intense THG signals of ordered lipid assemblies within $\mathrm{SC}^{29}$.

Melanin imaging based on its endogenous fluorescence was evidenced in 1979 by conventional one-photon excited fluorescence microscopy on human skin sections $s^{30}$ and 20 years later in vivo on forearm skin using twophoton excitation ${ }^{31}$. Multiphoton imaging enables in vivo human epidermis and superficial dermis characterization up to a depth of $\sim 200 \mu \mathrm{m}^{32,33}$. In addition to detecting intrinsic fluorescence from cellular and extracellular matrix constituents (e.g. keratin, $\mathrm{NAD}(\mathrm{P}) \mathrm{H}, \mathrm{FAD}$, melanin, and elastin) and second harmonic generation signals from fibrillar collagens ${ }^{34-36}$, multiphoton microscopy can be leveraged using fluorescence lifetime imaging (FLIM) to provide functional information on skin constituents ${ }^{37}$. Fluorescence lifetime is independent of the fluorophore concentration, but depends on the local microenvironment of the molecule, on variables such as $\mathrm{pH}$, binding status, and molecular conformational changes. The skin's autofluorescence lifetime (see ${ }^{38}$ and included references) spans from hundreds of picoseconds (e.g. melanin, free $\mathrm{NAD}(\mathrm{P}) \mathrm{H}$, bound $\mathrm{FAD}$ ) to nanoseconds (e.g. bound NAD $(\mathrm{P}) \mathrm{H}$, free FAD, keratin). Multiphoton FLIM imaging of melanin samples such as synthetic, Dopa or Sepia melanins, skin and eye melanocytes, human hair and hair bulb, and human skin (e.g. ${ }^{38-43}$ ) indicate a specific bi-exponential decay behavior with a predominantly ( $>90 \%$ relative contribution) short-fluorescence lifetime component around $\sim 100-200$ ps and a mixed species phasor plot with short phase lifetime distribution. However image acquisition time needed for acquiring correct fluorescence decays for bi-exponential or phasor $^{42-44}$ analysis is not compatible with $3 \mathrm{D}$ skin imaging in a clinical setup and in practice is limited to $2 \mathrm{D}$ imaging at selected epidermal depths.

Using in vivo 3D multiphoton imaging at $760 \mathrm{~nm}$ excitation, we were the firsts to propose an intensity-based melanin detection method. We compared it to histology Fontana-Masson and verified the 3D melanin global density modulation on human forearm skin upon topical application of corticosteroids in vivo ${ }^{45}$ and in vitro on reconstructed pigmented epidermis ${ }^{46}$. An intensity-based melanin detection upon $880 \mathrm{~nm}$ excitation was later used to characterize different skin color pigmentation phototypes ${ }^{47}$. This intensity-based approach works in the basal epidermal layers where melanin is highly concentrated and exhibits $2 \mathrm{PEF}$ signal intensities stronger than other endogenous fluorophores, but not in SC containing keratins with high fluorescence intensities. Moreover, pixels with low melanin concentration, low fluorescence intensity are not taken into account.

In order to detect melanin from multiphoton FLIM-like data compatible with 3D in vivo acquisitions on human subjects, we propose to use an approach combining (i) multiphoton FLIM, (ii) fast image acquisition times, and iii) a melanin detection method, that we call Pseudo-FLIM, which is based on slope analysis of the $2 \mathrm{PEF}$ intensity decay from temporally binned data ${ }^{48,49}$. In this paper, we validate our approach on synthetic melanin, melanocytes and keratinocytes coculture and in vivo human skin and compare it to FLIM bi-exponential and phasor analyses. Using parameters of melanin 3D global density and z-epidermal distribution, we assessed in vivo melanin modulations under different conditions: constitutive and acquired pigmentation, aging, natural UV exposure or application of topical retinoids known to having an effect on pigmentation.

\section{Materials and methods}

In vitro normal human melanocytes/keratinocytes (NHMK) coculture and synthetic melanin samples. Synthetic melanin (prepared by oxidation of tyrosine with hydrogen peroxide, SigmaAldrich M0418) dispersed in water was placed between a glass slide (VWR) and a glass coverslip (Marienfield $24 \times 50 \mathrm{~mm}^{2}$ ) using an adhesive silicon isolator (Grace Bio-Labs, JTR8R-0.5).

Normal human melanocytes/keratinocytes (NHMK) (see supplementary materials and methods) were grown in 96 well plates for 7 days.

In vivo human skin: clinical trials. All clinical studies were conducted in Paris, France (all volunteers gave written, informed consent and experimental protocols were approved by the Saint Louis Hospital ethics committee (EC), complying with the Declaration of Helsinki).

Constitutive pigmentation study (February-March 2010, EC reference 2010/01; original data). This study involved 37 female volunteers (18-55 y) with skin color determined by Individual Typology Angle (ITA): $>55^{\circ}$ (ITA group I, "very light skin", $n=4$ European origin (Eu.O) and $n=1$ Asiatic origin (As.O)); $41^{\circ}, 55^{\circ}$ ] (ITA group II, "light skin", $\mathrm{n}=5$ Eu.O and $\mathrm{n}=5$ As.O); $28^{\circ}, 41^{\circ}$ ] (ITA group III, “intermediate skin", $\mathrm{n}=5$ Eu.O and $\mathrm{n}=5$ As.O); $10^{\circ}, 2^{\circ}$ ] (ITA group IV, “tanned skin", $\mathrm{n}=4$ Eu. $\mathrm{O}, \mathrm{n}=2$ As.O and $\mathrm{n}=1$ African origin (Af.O)); $30^{\circ}$, $10^{\circ}$ ] (ITA group V, "brown skin", $\mathrm{n}=5$ Af.O). Multiphoton and colorimetry (Datacolor, Montreuil, France) measurements were performed on ventral forearm. This study allowed to determine the changes in melanin global density and z-epidermal distribution with both skin color and ethnicity. 
Photo-aging study (January-February 2009, EC reference 2008/62; parameters of melanin characterization are original data, other data from this study having already been published ${ }^{50}$,) This study involved 15 young (18-25 y) and 15 aged (70-75 y) female volunteers with Fitzpatrick phototypes I to IV. Imaging was performed on ventral and dorsal (mostly unexposed vs. exposed) forearms. Melanin modulations in this context revealed chronic cumulative sun exposure at two different ages.

Retinoids under occlusion study (March-July 2012, EC reference 2012/05; published ${ }^{51}$ ). This study involved 20 female volunteers (50-65 y) with skin color ITA between $10^{\circ}$ and $41^{\circ}$ (ITA group III/IV). Two products, Retinol $0.3 \%$ (RO, Retinol $0.3^{\oplus}$, L'Oréal product) and all-trans Retinoic acid $0.025 \%$ (RA, Retacnyl ${ }^{\oplus}$, Galderma) were applied under occlusion on dorsal forearm side for 12 days. A third untreated, occluded area was used as control. Imaging was performed at days D0, D12 (end of occlusion period), D18 and D32. This study aimed at studying effects of reference anti-aging products on melanin among other parameters in a short-term in vivo model.

Retinoids 1-year study (February 2011-April 2012, EC reference 2010/58; published $^{52}$ ) This study involved 30 female volunteers (50-65 y) with skin color ITA between $10^{\circ}$ and $41^{\circ}$ (ITA group III/IV). They applied Retinol $0.3 \%$ (RO, Retinol $0.3 \%$ cream, L'Oréal group) $(\mathrm{n}=15)$ or all-trans-Retinoic acid $0.025 \%$ (RA, Retinoic acid $0.025 \%$ cream, Galderma) $(n=15)$ on one dorsal forearm versus a control product (white paraffin containing excipient, Bayer) on the other forearm for 1 year. Imaging was performed at months M00 (March), M03 (June), M06 (September), M12 (March + 1 yr). This study aimed at studying effects of reference anti-aging products on melanin among other parameters in "real life" applications; acquisitions on the control conditions allowed to study melanin modulation with seasonality.

In vitro 2D multiphoton FLIM imaging. Multiphoton FLIM imaging (2D 2PEF FLIM) of synthetic melanin and NHMK coculture was performed with a commercial microscope (Leica TCS SP8 MP FLIM, Leica, Germany) integrating a time-correlated single photon counting (TCSPC) PicoHarp 300 module (Picoquant, Germany) and an $80 \mathrm{MHz}$ IR fs pulsed laser (Newport Chameleon Ultra II, 680-1080 nm). Images were acquired upon $760 \mathrm{~nm}$ excitation and detection in the $410-650 \mathrm{~nm}$ range using a $40 \times / 1.1 \mathrm{NA}$ water immersion objective. Images of $205 \times 205 \mu^{2}(512 \times 512$ pixels $\times 3128$ time channels; 4 ps/time channel, $0-12.5$ ns time range, position of the peak of the intensity decay at $1.33 \mathrm{~ns}$ ) were acquired with $1.2 \mu$ s pixel dwell time, 50 frame accumulation number at $2 \mathrm{~mW}$ for melanin solution, and respectively 200 for NHMK coculture at $5 \mathrm{~mW}$.

In vivo 2D multiphoton FLIM imaging. Imaging at different skin depths was performed using DermaInspect $^{\text {tix }}$ (JenLab GmbH, Jena, Germany) medical device integrating TCSPC detectors, a FLIM module (SPC830, Becker \& Hickl, Berlin, Germany) and an $80 \mathrm{MHz}$ IR fs pulsed laser (MaiTai Spectra-Physics, Mountain View, CA, USA). Images of $130 \times 130 \mu \mathrm{m}^{2}(511 \times 511$ pixels; $51 \mu$ s pixel dwell time $)$ were acquired upon $760 \mathrm{~nm}$ excitation and detection in the $390-650 \mathrm{~nm}$ range using a $40 \times / 1.3$ NA oil immersion objective (exponentially increased excitation power from $12 \mathrm{~mW}$ at the skin surface up to $47 \mathrm{~mW}$ at depths exceeding $75 \mu \mathrm{m}$ ). The signals from 4 pixels were combined during acquisition with SPCM software to give $127 \times 127$ pixels $\times 256$ time channels images ( $48.8 \mathrm{ps} /$ time channel, $0-12.5 \mathrm{~ns}$ time range, maximum intensity decay at $1.33 \mathrm{~ns} ; 13.4 \mathrm{~s} /$ image; $104 \mu \mathrm{s} /$ binned pixel).

In vivo 3D multiphoton imaging: 2PEF-FLIM (4 time channels)/SHG z-stacks. Multiphoton imaging (DermaInspect ${ }^{\mathrm{Tm}}$, Jenlab, Germany) was performed as previously described ${ }^{45}$. For each experimental condition, we acquired two adjacent $3 \mathrm{D}$ z-stacks (70 images; $2.346 \mu \mathrm{m}$ z-step). Image characteristics: $511 \times 511$ pixels $(0.255 \mu \mathrm{m} /$ pixel $) \times 4$ time channels $(2.08 \mathrm{~ns} /$ time channel), $0-8.33 \mathrm{~ns}$ time range (when acquiring 4 time channel images, the time range needed to be decreased from $12.5 \mathrm{~ns}$ to a software predefined value of $8.33 \mathrm{~ns}$ in order to achieve the $\sim 2 \mathrm{~ns}$ temporal binning for the Pseudo-FLIM analysis), maximum intensity decay at $1.33 \mathrm{~ns}$; $28 \mu$ s pixel dwell time; 7.4 s/image; $9.4 \mathrm{~min} / 3 \mathrm{D}$ z-stack.

FLIM bi-exponential analysis. FLIM parameters were computed by fitting the fluorescence intensity decay with a bi-exponential function ${ }^{38-41} I_{2 P E F}(t)=a_{1} e^{-\frac{t}{\tau_{1}}}+a_{2} e^{-\frac{t}{\tau_{2}}}$ to extract the short and long fluorescence lifetimes $\tau_{1}$ and $\tau_{2}$, their relative amplitudes $a_{1}[\%]=\frac{a_{1}}{a_{1}+a_{2}}$ and $a_{2}[\%]=\frac{a_{2}}{a_{1}+a_{2}}$ and compute amplitude-weighted $\tau_{A v A m p}=\frac{a_{1} \tau_{1}+a_{2} \tau_{2}}{a_{1}+a_{2}}$ or intensity-weighted $\tau_{A v \operatorname{Int}}=\frac{a_{1} \tau_{1}^{2}+a_{2} \tau_{2}^{2}}{a_{1} \tau_{1}+a_{2} \tau_{2}}$ average lifetimes. Simple fluorescent molecules have single exponential decays (e.g. simulated decays Fig. 1a), but in skin, the shape of this decay varies depending on the local microenvironment (e.g. $\mathrm{pH}$, binding status, molecular conformational changes) of the fluorophores and on the mixed ratio of fluorescent signals arising from free/bound $\mathrm{NAD}(\mathrm{P}) \mathrm{H}$, free/bound FAD, keratin, melanin, elastin, etc. Pixels with mostly melanin, as a main contributor to the detected signal, will exhibit a decay similar to the simulated A\&B mixed species decay (Fig. 1a).

FLIM bi-exponential analysis of in vitro $(512 \times 512$ pixels $\times 3128$ time channels $)$ and in vivo $(128 \times 128$ pixels $\times 256$ time channels) data is described in the supplementary materials and methods.

FLIM phasor analysis. By applying a Fourier transform to every pixel, phasor method ${ }^{44,53,54}$ transforms the $2 \mathrm{PEF}$ intensity decay into a phasor with coordinates $\mathrm{g}$ and $\mathrm{s}$ within the phasor plot (Fig. 1b, see details in Supplementary materials and methods).

In human skin, the $2 \mathrm{PEF}$ signal decays as a sum of multiple exponentials and is a mixture of two or more lifetime components originating either from two different molecules (e.g. free NAD $(\mathrm{P}) \mathrm{H}$ and bound NAD $(\mathrm{P})$ $\mathrm{H}$ ), a single molecule (e.g. melanin, elastin) or a mixture of molecules (e.g. melanin and free-/bound-NAD $(\mathrm{P}) \mathrm{H}$, keratin and free-/bound-NAD $(\mathrm{P}) \mathrm{H}$, etc.). Consequently, the phasor plot of human skin lies within the universal 
a)

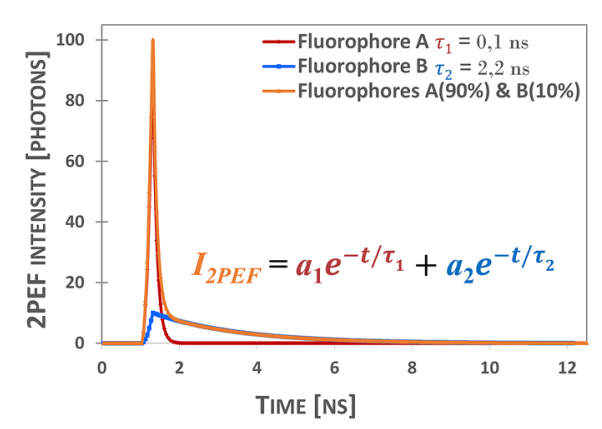

b)

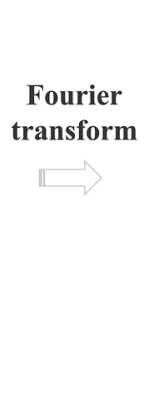

FLIM - Phasor plot

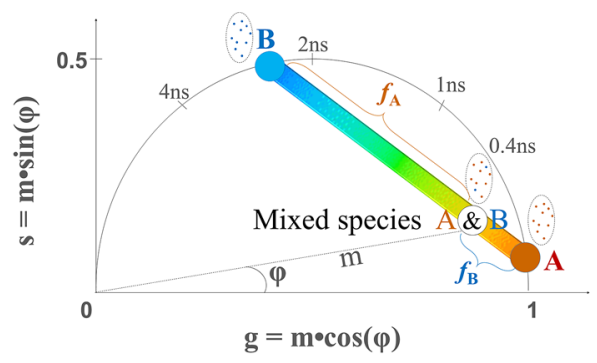

c) Pseudo-FLIM analysis: Temporal binning $\rightarrow$ Ln of 2 PEF Int. $\rightarrow$ Linear regression $\rightarrow$ Slope estimation

c1)

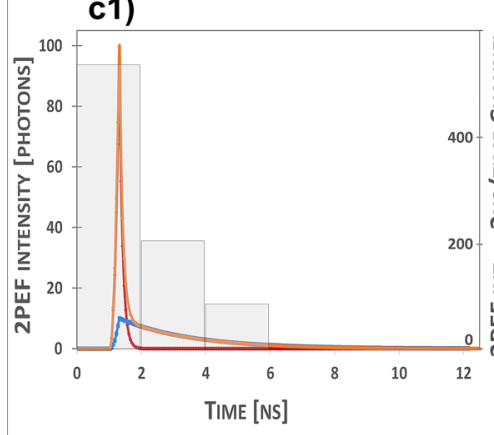

c2)

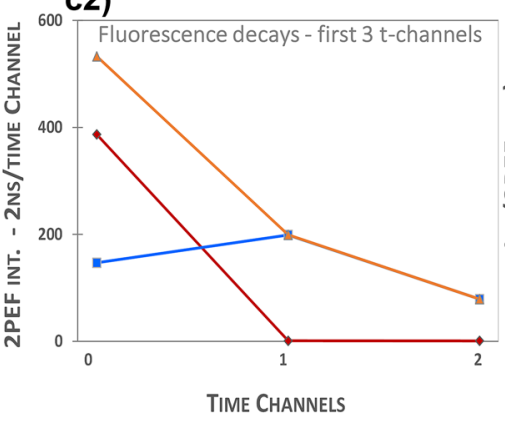

c3)

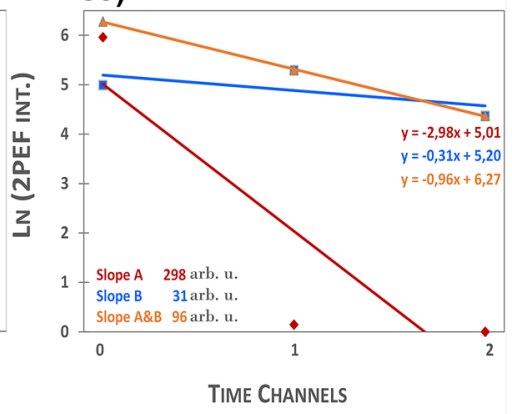

Figure 1. Principle of FLIM bi-exponential, Phasor and Pseudo-FLIM analyses. (a) Simulated monoexponential (fluorophores A and B) and bi-exponential (mixed species of fluorophores A and B with respectively $90 \%$ and $10 \%$ relative contribution) two-photon excited fluorescence intensity decays ( $12.5 \mathrm{~ns}$ time range; $80 \mathrm{MHz}$ ); this shape of $\mathrm{A} \& \mathrm{~B}$ bi-exponential decay can be measured in melanin containing samples. In FLIM bi-exponential analysis, the $2 \mathrm{PEF}$ intensity decay is adjusted with the function in (a) to compute the values of short- $\tau_{1}$ and long- $\tau_{2}$ fluorescence lifetimes and their relative contributions $a_{1}$ [\%] and $a_{2}$ [\%]. Images of FLIM bi-exponential fit and combination parameters such as amplitude- and intensity-weighted lifetimes are used for data analyses. (b) FLIM phasor analysis transforms a decay into a phasor with polar coordinates $g$, s, corresponding to the real and complex components of the Fourier transform, that can also be expressed as a function of $m$ modulation and $\varphi$ phase angle. Mono-exponential decays such as $\mathrm{A}$ and $\mathrm{B}$ will have their phasors on the semi-circle, whereas mixed species will have a phasor along a line connecting the two distinct lifetime phasors of $\mathrm{A}$ and $\mathrm{B}$. The relative fractions $f_{A}$ and $f_{B}$ can be computed from the distances of $\mathrm{A} \& \mathrm{~B}$ mixed species phasor to B and respectively A phasors. Images of $g$ and $s$ as well as combination parameters such as the apparent phase and modulation lifetimes and their relative fractions are used for data analyses. (c) Pseudo-FLIM analysis firstly involves a temporal binning of the $2 \mathrm{PEF}$ decay into a reduced number of time channels with $2 \mathrm{~ns}$ integration time per channel (see c1, gray bars indicate the photon intensity of the mixed A\&B species within the first 3-time channels with 2 ns binning). The $2 \mathrm{PEF}$ intensity of the binned first 3-time channels for simulated A, B and mixed A\&B species is given in (c2). After a natural logarithm transformation (c3), a linear regression of the first 3-time channels is performed to calculate the slope of the decay which is multiplied by a - 100 factor to create the Pseudo-FLIM slope parameter. The faster the decay, the higher the slope. Pseudo-FLIM image of the slope parameter is further processed for melanin detection by applying a threshold to keep the pixels with high slope values.

semicircle and reflects the mixed molecular species contributions. By observing the pixels clustering in the phasor plot and by mapping the clusters to their corresponding $i, j$ pixels within the FLIM image, skin layers or cells with similar phasors can be identified. One can also use the morphological/structural tissue information to attribute the phasor cluster to a specific skin constituent (e.g. elastin within the elastic fibers).

Phasor analysis was performed with SPCImage (v 8.1, Becker \& Hickl, Berlin, Germany) and home-written macro with Fiji/ImageJ (W. Rasband, NIH, USA). After bi-exponential analysis, g and s parameters were calculated using the same fixed shift parameter for all the images and phasor plot images were color $\operatorname{coded}$ for $\tau_{1}$ or $\tau_{2}$ fluorescence lifetimes. Exported $g$ and $s$ images were further analyzed with the Fiji macro to calculate the phase $\tau_{\varphi}$ and modulation $\tau_{m}$ lifetimes. All these images were further processed for melanin quantification with Fiji. Phasor plot color coded images were modified with Fiji to change the black background color to white.

Pseudo-FLIM analysis for 2D and 3D melanin detection. FLIM images with different number of time-channels and temporal binning can be analyzed by Pseudo-FLIM for melanin detection ${ }^{48,49}$ (see Fig. 1c). The main idea is to bin the fluorescence photons in a reduced number of time channels either at the acquisition or afterwards during processing and perform a linear regression to estimate the slope of the decay. FLIM 2PEF 
a)
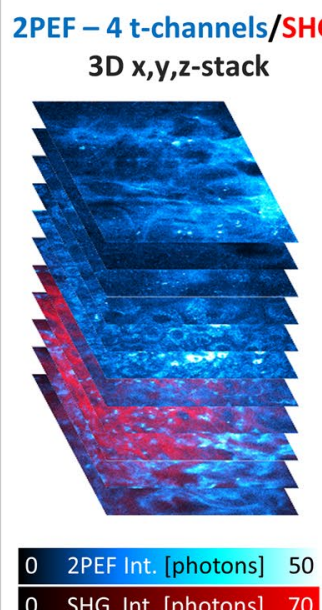

0 SHG Int. [photons] 70 $7,4 \mathrm{~s} /$ image
3D Multiphoton IMAgING - 2PEF /SHG \& Processing

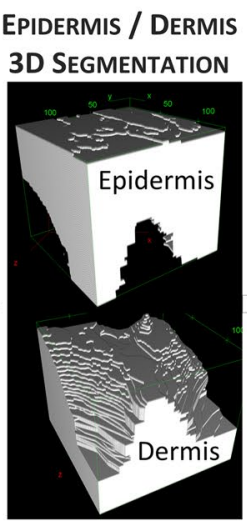

EPIDERMIS / DERMIS 3D QUANTIFICATION

3D MORPHOLOGY

- ED, SC, LED Thickness

- DEJ undulation

3D DENSITY

- Elastin

- Fibrillar Collagens

\section{b)}

- Raw 4-time channels 2PEF images

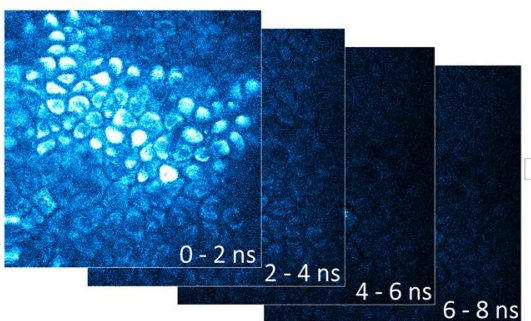

SC - stratum corneum, ED -epidermis, LED - living epidermis, DEJ-dermal-epidermal junction, $D$ - dermis

c)

\section{D EpIDermal Melanin QUantification}

3D Epidermal Melanin Density

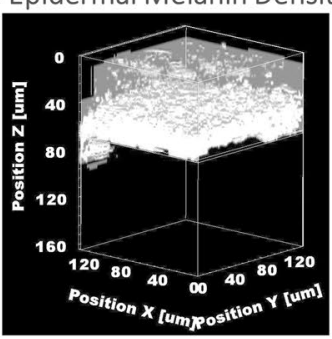

- Slopes parametric image

- Melanin mask

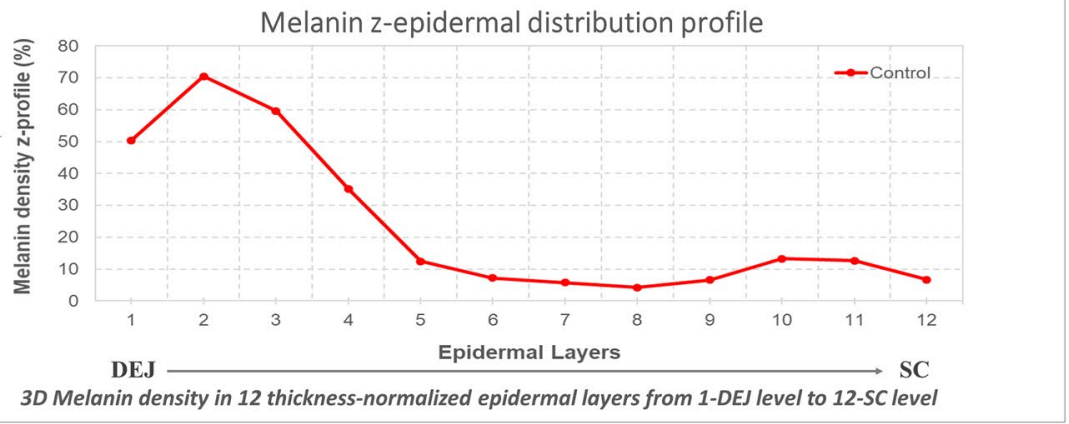

Figure 2. Global analysis process for in vivo 3D multiphoton images allowing 3D skin automatic layers segmentation and constituents quantification. (a) The first step of global 3D analysis of z-stacks of combined 2PEF-FLIM (4 time channels)/SHG images briefly consists in identifying the epidermal and dermal layers (3D automatic segmentation) and quantifying their morphology (thickness, DEJ 3D-shape) and the 3D dermal density of elastin and fibrillar collagens. (b) The z-stack of 2PEF-FLIM (4 time channels) images is further processed for melanin detection using Pseudo-FLIM method. The first 3-time channels are used for slope parametric image calculation and a melanin mask is obtained by applying a threshold to keep the high slopes values (above 70). (c) The 3D z-stack of melanin masks and the 3D automatic segmentation of the epidermis and its sub-layers are jointly used to estimate a 3D epidermal, SC or LED melanin density. By defining 12 -thickness normalized epidermal layers, the epidermal melanin density z-distribution (z-profile of melanin density in the 12 thickness-normalized epidermal layers from 1-DEJ level to 12-SC level) can be assessed. 
intensity decays with a peak position (maximum of $2 \mathrm{PEF}$ intensity decay) at $1,33 \mathrm{~ns}$ are temporally binned into channels with 2 ns per time channel (Fig. 1c1). The first 3 time-channels are used for further analysis. Figure 1c2 shows the simulated $2 \mathrm{PEF}$ intensity decays with 2 ns temporally binned channels of fluorophores A, B and mixed species A\&B. Mono-exponential short-fluorescence lifetime decays (e.g. A, $0.1 \mathrm{~ns}$ ) will have a higher 2PEF intensity in the first binned time-channel compared to long-fluorescence lifetime decays (e.g. B, $2.2 \mathrm{~ns}$ ). The photons in this first binned time-channel are an integral of noise photons (detected before the rising edge) and fluorescence photons. The higher the photon intensity within this 2 ns time window, the higher the slope of the decay (see Fig. 1c2 and c3). The decays are transformed in $\ln (2 \mathrm{PEF}$ Intensity) and a linear regression is performed to extract the slope of the decay (Fig. 1c3). In this example, fluorophore A $\left(\tau_{1}=0.1 \mathrm{~ns}\right)$ has a higher slope compared to fluorophore B $\left(\tau_{2}=2.2 \mathrm{~ns}\right)$. Mixed species A\&B decay with $90 \%$ contribution of A has also a high slope compared to B, but smaller than A. In tissues, the difference between different mixed species decays will come from differences in both fluorescence lifetimes and in their relative contributions. Fast decays with high relative contributions will have high Pseudo-FLIM slopes.

Figure 1 illustrates Pseudo-FLIM analysis at the pixel level. When analyzing images, we first calculate the temporal binning and apply a Gaussian blur with a radius $r=\sqrt{\frac{\text { Melaninfilterarea/d } x^{2}}{\pi}}$ where $d x$ is the pixel size in $\mu \mathrm{m}$ and melanin filter area was fixed to a value of $1 \mu \mathrm{m}^{2}$ proximal to the melanosome area. As Pseudo-FLIM melanin analysis requires only few photons per pixel (see "Results"section), it results in faster image acquisition compatible with 3D imaging on humans. After temporal binning and Gaussian blurring, some pixels in the 3D z-stack of 2PEF FLIM ( 4 time channels) images will have zero values (images acquired above the skin surface, sometimes pixels within less or non-fluorescent regions such as nuclei and cellular membranes). Before calculating the natural logarithm, we add 1 photon to all the pixels of the temporally binned images. A Pseudo-FLIM slope parametric 8 bits image is created by multiplying the slope of the decay (results of the linear regression fit; mostly negative values) by a factor of -100 to have positive values and the pixels with values above 255 are set to 255 and below 0 to 0 . We express the Pseudo-FLIM slope parameter in arbitrary units, but one could also divide its value by the time channel duration and express it in 1/ns units (as it is done for single exponential decays: the decay time $\tau$ can be calculated from the slope of a plot of $\log I(t)$ versus $t$, or from the time at which the intensity decreases to $1 / e$ of the intensity at $t=0$ and the slope $=-1 / \tau)^{55}$.

Pseudo-FLIM slope parametric images are further processed for melanin mask calculation by applying a threshold to keep the high slope values pixels (above 70) mainly corresponding to melanin (see "Results"). We also apply an open area filter to remove the isolated regions of interest with an area smaller than the approximated $1 \mu \mathrm{m}^{2}$ melanosomal area. The influence of the temporal binning and of the maximum intensity peak position on Pseudo-FLIM results is described in supplementary materials and methods (Fig. S1).

We implemented Pseudo-FLIM analysis with both Python in Multiphoton Skin Tools Suite (MPSTS) software $^{50}$ and using a home-written macro with Fiji/ImageJ.

In vivo 3D automatic skin layers segmentation and constituents quantification. The global 3D analysis of z-stacks of combined 2PEF-FLIM (4 time channels)/SHG images was performed with MPSTS software ${ }^{50}$ to identify skin layers, characterize DEJ 3D-shape and extract quantitative parameters on skin constituents and layers (Fig. 2; see Supplementary materials and methods).

Statistical analysis. The results are mainly given in the text as estimated mean and standard deviation of the mean and the data distributions are described using histograms or box plots. Clinical trials data have been interpreted using both effect size (ES) and inferential analysis as currently recommended ${ }^{56}$. The data number per group is twice the number of volunteers as the two adjacent $3 \mathrm{D}$ z-stacks acquired per volunteer were considered separately.

\section{Results}

Comparison of FLIM and Pseudo-FLIM methods for melanin detection in different in vitro samples. Two-photon excited fluorescence intensity decays of synthetic melanin and normal human melanocytes/keratinocytes (NHMK) coculture were analyzed with FLIM and Pseudo-FLIM methods (Fig. 3). As expected, FLIM analysis allowed evidencing that synthetic melanin samples (Fig. 3a) have a short fluorescence lifetime $\tau_{1}$ of $66 \pm 14$ ps (see also its histogram in Fig. 3c) with a main relative contribution $a_{1}$ [\%] of $99 \pm 2 \%$ and a long fluorescence lifetime $\tau_{2}$ of $1.59 \pm 1.82 \mathrm{~ns}$. These values are statistics over all the pixels within 5 images of $205 \times 205 \mu \mathrm{m}^{2}(5 \times 512 \times 512$ pixels $)$ acquired at different locations within the synthetic melanin solution. This type of melanin is characterized by a fast decay, as shown in the histogram of photons arrival times in Fig. 3c and Pseudo-FLIM analysis yields indeed high slope parameter values of $178.75 \pm 9.69 \mathrm{arb}$. u. We found similar fast decays and high slopes for natural melanins such as Sepia melanin and excreted melanin in 2D melanocytes cultures (data not shown). In NHMK coculture, we found two sets of pixels (e.g. for Fig. $3 \mathrm{~b}: \tau_{1}=53 \pm 14$ ps; $a_{1}$ [\%] $=96 \pm 3 \% ; \tau_{2}=2.31 \pm 0.64 \mathrm{~ns}$ and respectively $\tau_{1}=419 \pm 148 \mathrm{ps} ; a_{1}[\%]=60 \pm 9 \% ; \tau_{2}=2.73 \pm 0.30 \mathrm{~ns}$, statistics over the pixels in Fig. 3b) characterized by either (i) high slopes and FLIM lifetimes and relative contributions parameters similar to synthetic melanin or (ii) small slopes and FLIM parameters similar to free $\mathrm{NAD}(\mathrm{P}) \mathrm{H}$ and bound FAD and respectively bound $\mathrm{NAD}(\mathrm{P}) \mathrm{H}$ and free FAD (see Fig. 3b, $\mathrm{d}$ and e). The fluorescence lifetimes of NAD(P)H and FAD are exquisitely sensitive to enzyme binding during the cycling of the electron transport chain: proteinbound $\mathrm{NAD}(\mathrm{P}) \mathrm{H}$ lifetime is significantly longer than the free $\mathrm{NAD}(\mathrm{P}) \mathrm{H}$ lifetime, due to self-quenching in the free state while the FAD lifetime is short and long in the protein-bound and free states, respectively ${ }^{57-59}$. We recently demonstrated in unpigmented reconstructed human skin that single wavelength excitation at $760 \mathrm{~nm}$ enables both $\mathrm{NAD}(\mathrm{P}) \mathrm{H}$ and FAD imaging in keratinocytes, but the resulting $2 \mathrm{PEF}$ FAD intensity is low due 
FLIM analysis $\rightarrow$ Raw 2PEF FLIM 512x512 x 3128 t-channels; Max[I(t)]: 1,33ns; 4ps/t-channel

a)

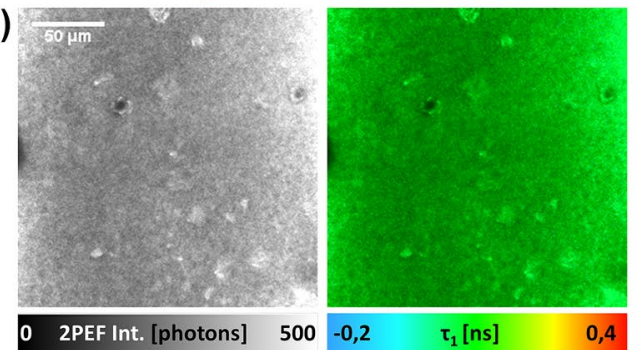

b)

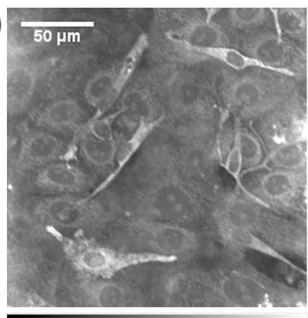

2PEF Int. [photons] 5000
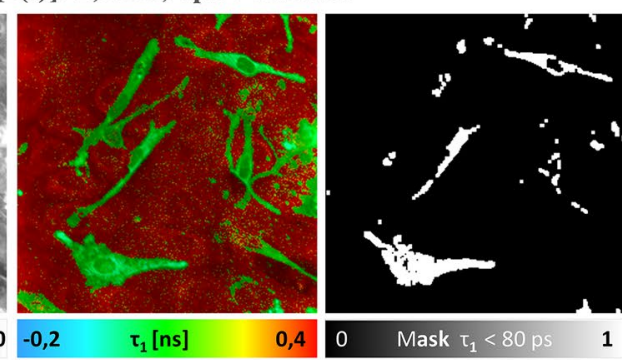

c)

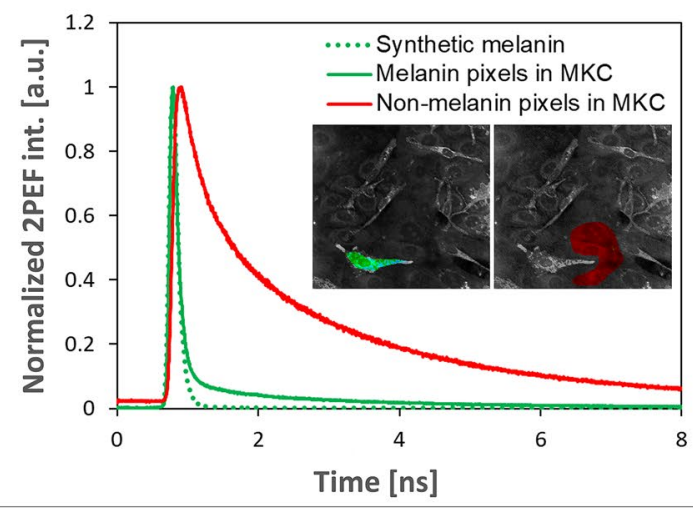

d)

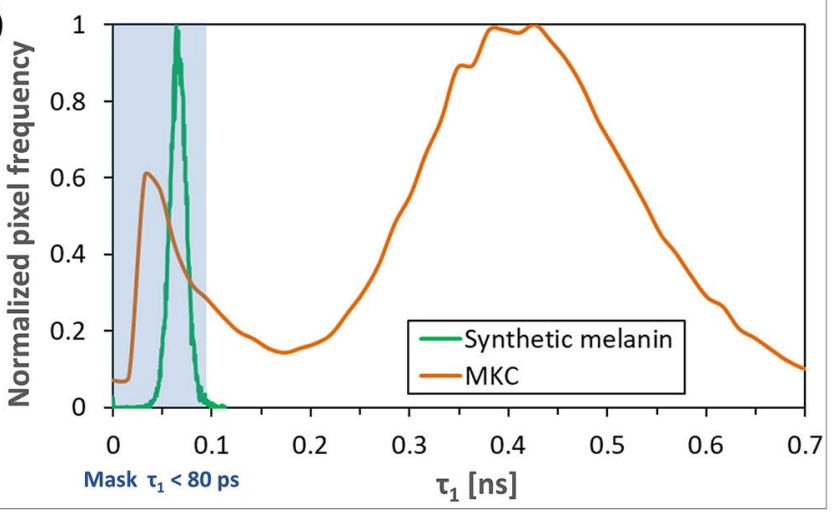

e) Pseudo-FLIM analysis $\rightarrow$ Temporal binning 2PEF FLIM 512x512 x 4 t-channels; Max[I(t)]: 1,33ns; 2ns/t-channel

1] 2 ns - temporal binning - First 3-t channels

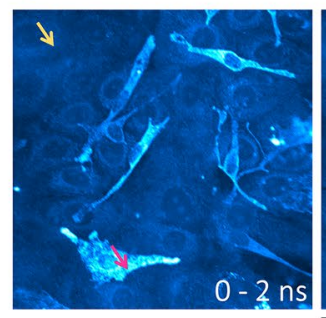

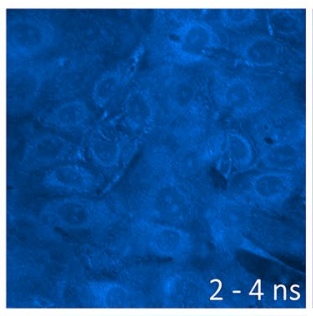

2PEF Int. [photons] 1000
2] Slope parametric image \& Melanin mask
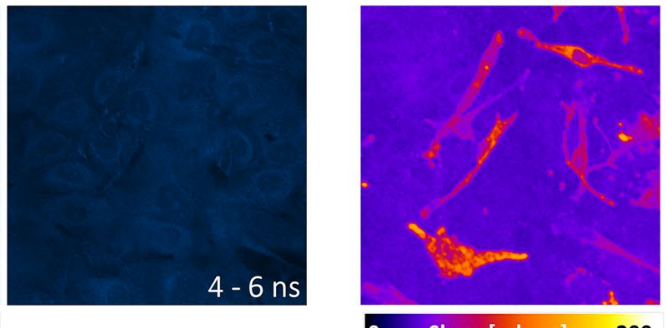

0 Slope [arb. u.] 200

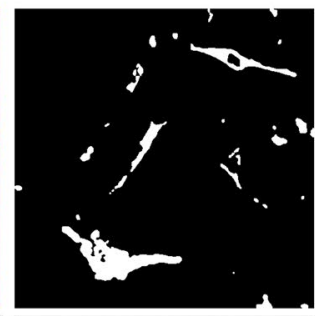

Slope Melanin Mask 1 f)

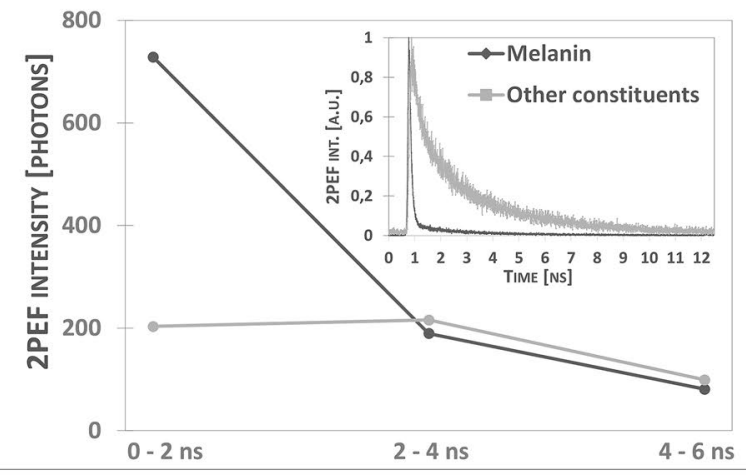

g)

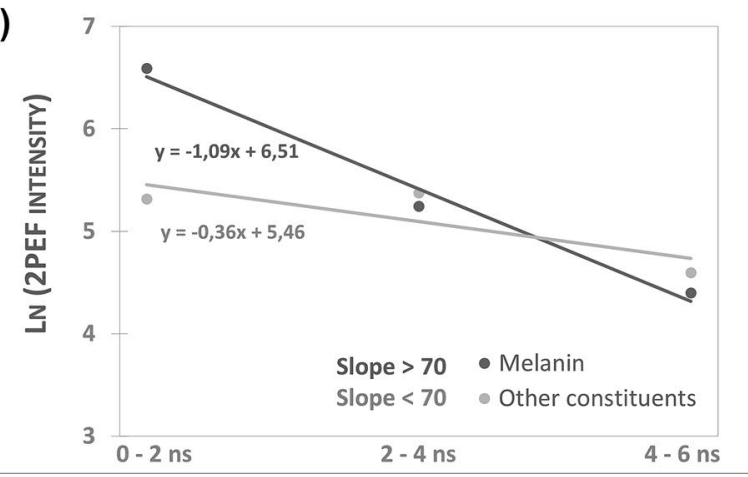

Figure 3. Comparison of FLIM bi-exponential fitting and Pseudo-FLIM methods for melanin detection. FLIM analysis - 2PEF intensity and $\tau_{1}$ short-fluorescence lifetime images of (a) synthetic melanin and (b) normal human skin melanocytes and keratinocytes NHMK coculture. The FLIM $\tau_{1}$ - based melanin mask is obtained by application of a threshold to keep the pixels with $\tau_{1}$ values below 80 ps. (c) $2 \mathrm{PEF}$ intensity decays of synthetic melanin, melanin and non-melanin pixels within the NHMK coculture (averaged 2PEF intensity within the green and respectively red regions of interest). (d) Normalized histograms of the pixel frequency for $\tau_{1}$ FLIM images of synthetic melanin (a) and NHMK coculture (b). The blue rectangle depicts the histogram regions selected by the application of a $\tau_{1}<80$ ps threshold. (e) Pseudo-FLIM analysis of NHMK coculture, involving (1) a temporal binning of the $2 \mathrm{PEF}$ decay into a reduced number of time channels with 2 ns integration time per channel, followed by (2) an estimation of the slope of the decay after linear regression on the first $3 \mathrm{t}$-channels. (f) Example of resulting binned 2PEF decay of a melanin pixel (red arrow) and a nonmelanin pixel (yellow arrow) selected based on their $\tau_{1}$ value. (g) After a natural logarithm transformation, a linear regression of the first 3-time channels is performed to calculate the slope of the decay. The faster the decay, the higher the slope, as observed for these two melanin and non-melanin pixels. The image of the Pseudo-FLIM slope parameter in (e) is further processed for melanin detection to keep the high slopes values above 70 arb. u. and create a Pseudo-FLIM melanin mask. 
to the disparities in the coenzymes's concentration ${ }^{60}$, thus the $2 \mathrm{PEF}$ intensity signal at $760 \mathrm{~nm}$ in our cell samples mainly arises from $\mathrm{NAD}(\mathrm{P}) \mathrm{H}$ and melanin. Figure $3 \mathrm{~d}$ shows the histogram of $\tau_{1}$ with values up to 200 ps mostly detected in melanocytes and in some pixels within keratinocytes, indicating the melanin transfer from melanocytes to keratinocytes. These values are in agreement with the reported $\tau_{1}$ values for melanin containing samples such as Dopa melanin and black hair ${ }^{39}$, melanocytes investigated in melanocytic nevi and melanomas ${ }^{40}$, melanocytes in a 3D skin model, human skin basal layer and hair bulb ${ }^{41}$ or synthetic melanin, melanocytes/ keratinocytes coculture and human $\operatorname{skin}^{38}$. Pseudo-FLIM analysis of NHMK coculture is detailed in Fig. 3e, $\mathrm{f}$ and $\mathrm{g}$. The fast decays, fast decrease in melanin 2PEF signal intensity with time is clearly visible on the first 3 t-channels images with 2 ns temporal binning (Fig. 3e). In Fig. $3 \mathrm{f}$ we have plotted both the raw decays (inserted graph) and the temporally binned decays for 2 pixels chosen within respectively melanocytes and keratinocytes cells. These pixels were attributed to melanin and respectively other endogenous constituents $(\mathrm{NAD}(\mathrm{P}) \mathrm{H}$ and FAD) based on their $\tau_{1}$ values and present different slope values (see Fig. $3 \mathrm{~g}$ ).

Based on these findings, we sought to define thresholds for FLIM short-fluorescence lifetime and PseudoFLIM slope parameter that will enable melanin detection in these samples. For synthetic melanin, based on the histogram in Fig. 3 d, a $\tau_{1}<80$ ps threshold will allow selecting all melanin pixels. This type of synthetic melanin characterized by a predominant $(\sim 99 \%)$ and very short $(<80 \mathrm{ps})$ fluorescence lifetime and a long fluorescence lifetime $(\sim 1.59 \mathrm{~ns})$ results in a very fast 2PEF intensity decay (Fig. 3c), corresponding in Pseudo-FLIM analysis to very high slopes values. Thus, for this type of "pure" melanin sample, a Pseudo-FLIM melanin mask could be obtained by applying a threshold on the slope parametric image to keep the slopes above $100 \mathrm{arb}$. $\mathrm{u}$. In the melanocyte/keratinocyte coculture, the FLIM parameters are slightly different compared to the ones of synthetic model melanin and this is expected as natural melanin is a mixture of eu-/pheo-melanin ${ }^{6}$. Modulations in these $\tau_{1}, \tau_{2}, a_{1}[\%]$ or $a_{2}[\%]$ parameters will be reflected on the shape of the 2PEF intensity decay and thus on the steepness of the slope of the decay. This is clearly seen in Fig. $3 \mathrm{c}$ for the melanin containing pixels in the melanocyte ( $\tau_{1} \sim 53 \mathrm{ps}, a_{1}[\%] \sim 96 \%$ and $\tau_{2} \sim 2.3 \mathrm{~ns}$ ). The changes in these parameters, and mainly the increase in $\tau_{2}$, results in slightly slower decays, corresponding to smaller Pseudo-FLIM slope values as compared to synthetic melanin. Accordingly, the threshold value for Pseudo-FLIM slope parameter must be decreased. We found that by applying a threshold to keep the high slope values above 70 arb.u., the Pseudo-FLIM melanin masks highlights almost the same pixels as the ones in the FLIM $\tau_{1}<80$ ps melanin mask (Fig. $3 \mathrm{a}$ and e). These data allow demonstrating that Pseudo-FLIM analysis can identify melanin pixels based on the slope of their 2PEF intensity decay in synthetic melanin and melanocytes keratinocytes coculture.

Comparison of FLIM, phasor and Pseudo-FLIM methods for melanin detection in human skin in vivo. Next, we sought to determine if the same threshold for Pseudo-FLIM melanin mask defined in the NHMK coculture also works for in vivo human skin. We have acquired 2D 2PEF FLIM images at different depths from the skin surface to the dermis, within SC disjunctum, SC compactum, granulosum, spinosum, basale and superficial dermis and analyzed the data with FLIM bi-exponential fitting, phasor and Pseudo-FLIM methods (Fig. 4, Fig. S2). Our imaging conditions (760 nm excitation, broad spectral detection 390-650 nm) were chosen to detect the endogenous autofluorescence of skin constituents such as $\mathrm{NAD}(\mathrm{P}) \mathrm{H}, \mathrm{FAD}$, melanin, keratin and elastin $^{35,36,42}$. The diversity in skin constituents and mixture of constituents at the pixel level is clearly seen in the phasor plots patterns of the different layers and bi-exponential fitting images. The histograms of parameters shown in Fig. 4 and their average values within different skin layers is given in Fig. S3.

Living cells such as non-melanized keratinocytes of the basal, spinosum and granulosum layers have fluorescence signals dominated by $\mathrm{NAD}(\mathrm{P}) \mathrm{H}$ 's emission in our imaging conditions ${ }^{61,62}$. Although FAD has a larger two-photon absorption cross section than $\mathrm{NAD}(\mathrm{P}) \mathrm{H}$ at $760 \mathrm{~nm}^{61}$, its low fluorescence quantum yield and the disparities in NAD $(\mathrm{P}) \mathrm{H}$ and FAD coenzymes's concentration lead to a low 2PEF FAD intensity, as measured in living keratinocytes within unpigmented reconstructed human skin ${ }^{60}$.The cells of stratum granulosum in Fig. 4 show a phasors's pattern and bi-exponential analysis parameters equivalent to the ones of living keratinocytes within unpigmented reconstructed human $\operatorname{skin}^{60,63}$.

Depending on the constitutive skin pigmentation, keratinocytes of the basal and supra-basal layers also contain different amounts of melanin within the melanosomes transferred from the melanocytes. Some melanin, may also be found in corneocytes, within non-degraded melanosomes. In normal human skin, melanocytes are found within stratum basale, but they cannot be identified in multiphoton FLIM based on their melanin content like in melanoma ${ }^{40,64}$ or in a $2 \mathrm{D}$ cellular culture (e.g. Fig. 3). Indeed, in healthy skin, fully melanized type IV melanosomes are found at the tips of their dendrites and are directly transferred to keratinocytes. Melanized keratinocytes have a shorter $\tau_{1}$ fluorescence lifetime (Fig. 4) and a higher relative amplitude $a_{1}$ [\%] (Fig. S2) compared to non-melanized keratinocytes with mainly $\mathrm{NAD}(\mathrm{P}) \mathrm{H}$ as a fluorescent constituent (e.g. $\tau_{1}$ and $a_{1}[\%]$ histograms in Fig. S3). These results are in agreement with human skin basal layer data published by Dancik et al. ${ }^{38}$. The shortest $\tau_{1}$ and highest $a_{1}[\%]$ values were mainly found within melanized keratinocytes in stratum basale and also within some keratinocytes and corneocytes within other skin layers. The phasor plots of stratum spinosum and basale, with a comet like pattern pointing towards a very short lifetime component around $0.1 \mathrm{~ns}$, are a mixture of different lifetime components (mainly free/bound $\mathrm{NAD}(\mathrm{P}) \mathrm{H}$, to a less extent free/bound FAD and melanin (short and long lifetimes)). Similar phasor pattern was also evidenced in human choroidal melanocytes (NADH/melanin constituents) ${ }^{43}$. The signal intensity was not high enough to allow for multi-exponential analysis, so the data were only approximated using a bi-exponential decay function. Pixels with high g and small $s$ values, corresponding to very small phase lifetimes, mainly contain melanin.

Pixels with high g and small s values, short phase lifetime, were also found within the capillary vessels of the dermis. In the example of Fig. 4, we detected a very short and predominant fluorescence lifetime component similar to melanin, probably arising from hemoglobin within the red blood cells ${ }^{65-67}$. Elastin within elastic fibers 

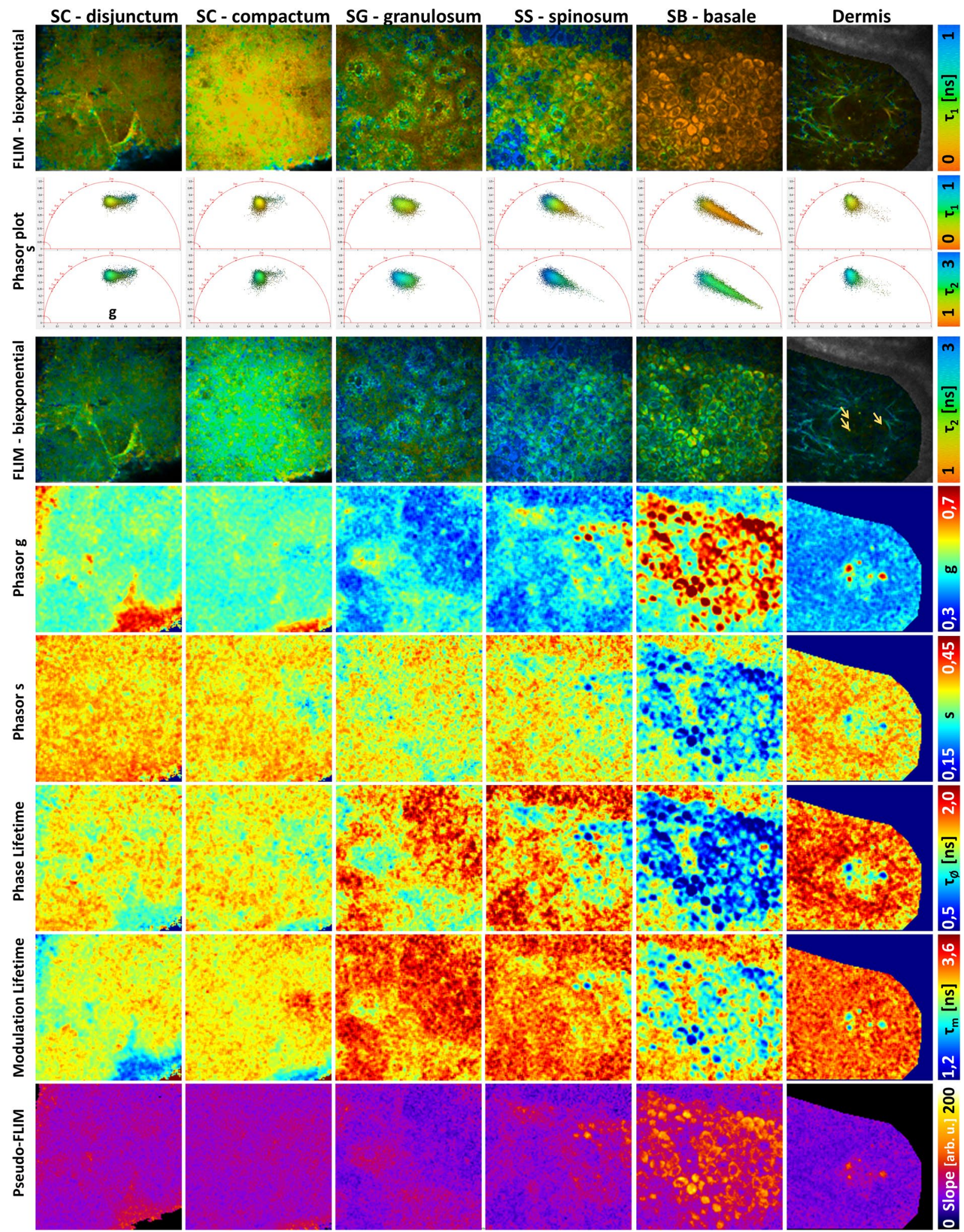

Figure 4. Comparison of FLIM bi-exponential fitting, Phasor and Pseudo-FLIM methods for melanin detection in vivo on human skin. Multiphoton 2D 2PEF FLIM images acquired at different depths from the skin surface to the dermis, within stratum cornuem (SC) disjunctum, corneum compactum, granulosum (SG), spinosum (SS), basale (SB) and superficial dermis were analyzed with the three methods. (top) FLIM bi-exponential fitting analysis images of the short $\tau_{1}$ and long $\tau_{2}$ fluorescence lifetime parameters. (middle) Phasor analysis images of $\mathrm{g}$, $\mathrm{s}$, phase lifetime and modulation lifetime parameters. The corresponding phasor plots ( $\mathrm{s}$ versus g scatters) of the different skin layers are inserted in between the $\tau_{1}$ and $\tau_{2}$ images and color coded using the same color scale as for $\tau_{1}$ and $\tau_{2}$ parameters. An enlarge view is shown in Fig. S2. (bottom) PseudoFLIM analysis images of the slope parameter highlighting pixels with a fast decay. The arrows indicate the fast decay pixels within the blood capillary. 
has longer phase lifetimes compared to melanin and a phasor plot overlapping with the one of non-melanized keratinocytes.

In $S C$, pixels with $\tau_{1}$ values around 250 ps and respectively 800 ps were found (Fig. S3). The long lifetime values could translate either the keratinization process or melanin degradation. We don't know how the natural process of melanin degradation in the upper skin layers impacts its molecular structure and organization and thus its fluorescence lifetime. The phasor plot pattern is also different compared to other layers: a round shape pattern shifted towards higher g values, although partly overlapping the phasors of non-melanized keratinocytes and a comet like pattern probably corresponding to more keratinized cells. The pixels at the tip of the comet are found within the uppermost corneocytes at the air/skin interface and are characterized by a major contribution of $\sim 800$ ps lifetime component. This phasor pattern is however different from the reported hair keratin phasor plot $^{42,43}$ suggesting different fluorescence properties of keratinized hair and skin tissues. Whether these pixels correspond to "more keratinized" structures or to degraded melanin it is unknown, and this will require further analyses, beyond the scope of this paper.

Pseudo-FLIM analysis highlights pixels with high slope values corresponding to fast 2PEF intensity decays. The images in Fig. 4 show that these high slope values pixels are also characterized by small $\tau_{1}$, high $a_{1}[\%]$, high g, small phase and modulation lifetimes values. Given all these skin characteristics, the question is how to quantify melanin from multiphoton FLIM data? We applied different thresholds to create melanin masks (Fig. S4): $\tau_{1}$ $<100 \mathrm{ps}, \tau_{1}<150 \mathrm{ps}, a_{1}[\%]>90 \%, \tau_{A v A m p}<400 \mathrm{ps}, \mathrm{g}>0,5 \& \mathrm{~s}<0.3$ (to keep the melanin containing pixels and remove the non-melanized pixels and the pixels within the comet pattern of the stratum corneum) and $\tau_{\varphi}<1,1 \mathrm{~ns}$. By applying a threshold to keep the high slope values above 70, we created a Pseudo-FLIM melanin masks similar to the melanin masks of amplitude weighted average lifetime (bi-exponential analysis) and phase lifetime (phasor analysis). Pseudo-FLIM mask includes the pixels within the $\tau_{1}<100$ ps and $a_{1}[\%]>90 \%$ masks but also includes other pixels. By increasing the threshold value of the short-fluorescence lifetime to $150 \mathrm{ps,}$ more pixels with smaller relative contributions are identified, but they are not selected by the other parameters.

Within SC, it seems that the slope $>70$ arb. u. threshold also allows identifying pixels with high g values, within the comet pattern. We also compared Pseudo-FLIM analysis (slope estimation upon linear regression on the first 3 temporally binned 2 ns time-channels, $1,33 \mathrm{~ns}$ peak position $)^{48,49}$ with an analysis based on the subtraction of two overlapping temporally binned time-channels ${ }^{68}$ that is a linear regression on two channels, corresponding to a Pseudo-FLIM analysis on two time-channels. The images (data not shown) were quite similar, and we observed that this 2 -channel method also detects the same pixels with high $g$ values, $\tau_{1} \sim 800 \mathrm{ps}, a_{1}[\%]$ $\sim 70 \%$, within the comet pattern of SC.

The comparison of FLIM bi-exponential, phasor and Pseudo-FLIM analyses allows confirming that in vivo melanin pixels characterized by $\tau_{1}<100 \mathrm{ps}, a_{1}[\%]>90 \%, \mathrm{~g}>0.5 \& \mathrm{~s}<0.3$ are detected by Pseudo-FLIM analysis, which also includes other pixels with $\tau_{\varphi}<\sim 1.1 \mathrm{~ns}$. Based on these considerations, melanin detection by PseudoFLIM seems to be specific within the living epidermis, but some fast decay pixels within some regions of the uppermost cells of SC are also detected.

Pseudo-FLIM melanin analysis is compatible with 3D imaging in clinical trials. We first acquired an in vivo multiphoton z-stack of combined 2PEF-FLIM (4 time channels)/SHG images with $28 \mu$ s pixel dwell time (Fig. 5b). We chose afterwards a 2D plane within the basal layer and acquired a 2PEF-FLIM image (256 time channels) with decreased spatial resolution ( $4 \times$ spatial binning of $511 \times 511 \rightarrow 127 \times 127$ pixels) and increased image acquisition time ( $104 \mu$ s pixel dwell time) (Fig. 5a). The two images were acquired almost within the same $\mathrm{z} 2 \mathrm{D}$ plane of the basal layer (a little shift in $\mathrm{z}$ can be perceived between images). The comparison of $2 \mathrm{~ns}$ temporally binned images clearly shows that the high intensity melanin signals are mostly decaying within the first 2 ns time-window. Figure $5 \mathrm{~b} 1$ and b2 show $2 \mathrm{PEF}$ intensity decays for a melanin pixel (fast decay, high slope value) and a "other constituents" pixel (slower decay, small slope value). After natural logarithm transformation and calculation of the linear regression (Fig. $5 \mathrm{c} 1$ and $\mathrm{c} 2$ ), we obtained almost the same slopes for melanin pixels. The slope parametric images, their histograms (Fig. $5 \mathrm{~d} 1$ and $\mathrm{d} 2$ ) and corresponding melanin masks (Fig. 5e1 and e2) are quite similar given the slight $z$-difference between the two images and the spatial resolution differences.

We can conclude that Pseudo-FLIM melanin detection is still feasible upon decreasing the image acquisition time and opens the possibility to quantify melanin from 3D z-stacks. An example of Pseudo-FLIM melanin detection from 3D multiphoton clinical data is given in Fig. 6. In this z-stack, one clearly can see that Pseudo-FLIM not only detected high 2PEF intensity pixels of basal keratinocytes but also pixels with lower 2PEF intensity but fast decays.

Applications of Pseudo-FLIM in vivo: modulation of melanin global 3D density and z-epidermal distribution upon different conditions. Measurement of melanin 3D density was performed in the entire epidermis (global density) and into several thickness-normalized epidermal sub-layers (z-distribution), allowing distinguishing its neosynthesis or inhibition in the basal layer versus modulations of its distribution in the upper layers. Changes in melanin density in epidermal sub-layers can impact or not the global density. For example, a decrease in one layer could be counteracted by an increase in another layer, and hence, no change will be evidenced in the global density. It also allows evidencing slight modulations that are not detectable at the global level ${ }^{52}$.

Constitutive pigmentation (original data). Combined 2PEF intensity and Pseudo-FLIM melanin masks images allow observing the changes in melanin density with skin color ITA groups (Fig. 7a). As expected, global 3D epidermal melanin density progressively increases in the lightest to darkest skin color groups (Fig. 7b, c, Table S1) and is highly correlated with skin ITA value (negatively, Fig. 7d) in agreement with ex vivo results 
a 1) Raw 2PEF FLIM 127x127x256 t-channels; Max[I(t)]: 1,33ns; 48,8ps/t-channel; 13,4s/image Raw 2PEF Int. $I=\sum I_{i} \quad$ 1] 2 ns - temporal binning $\rightarrow 4$ t-channels data
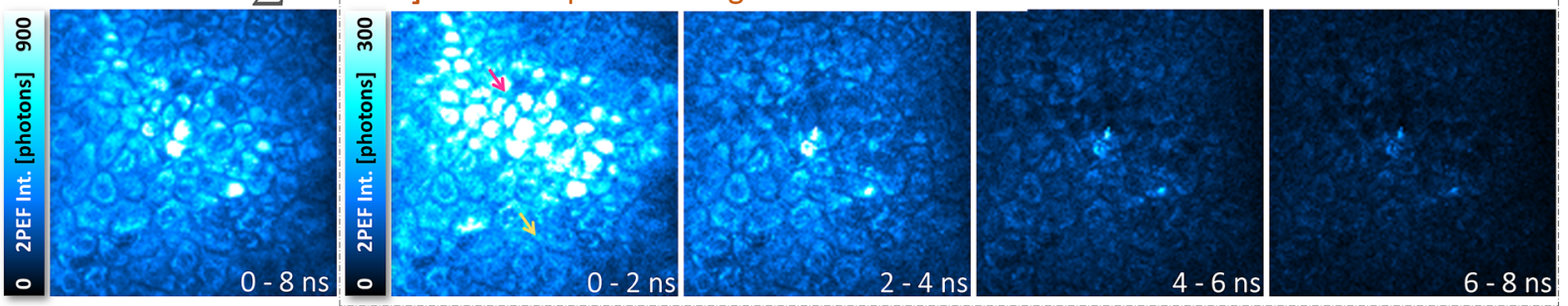

$a_{2}$ ) Raw 2PEF FLIM 511x511x4 t-channels; Max[I(t)]: 1,33ns; 2ns/t-channel; 7,4s/image

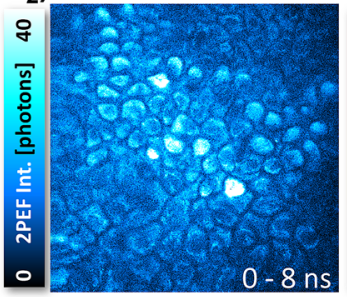

$\left.b_{1}\right)$

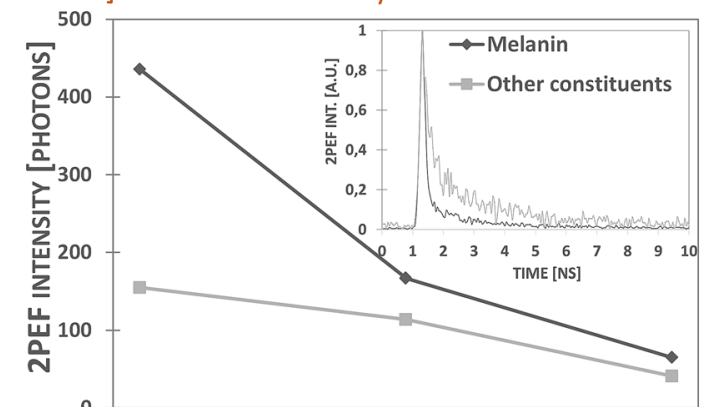

$b_{2}$ )

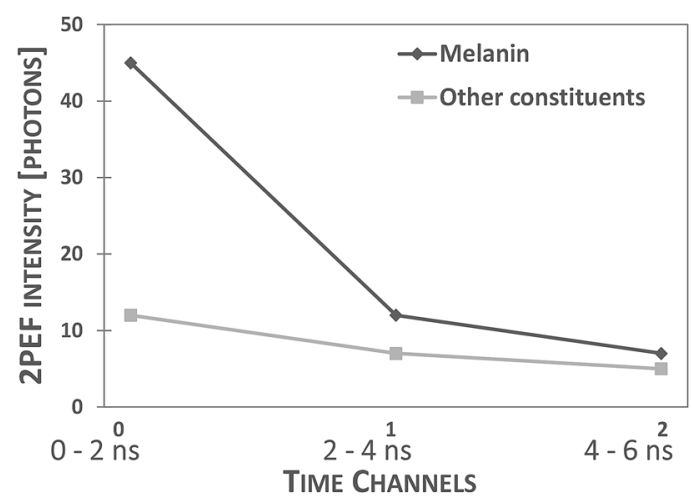

4] Slope parametric images
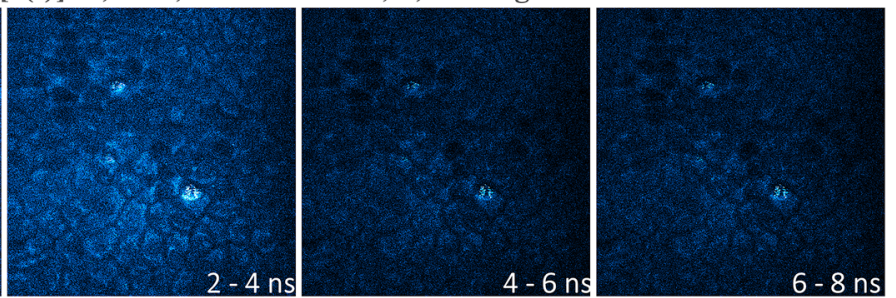

$\left.c_{1}\right)$

3] Ln of 2PEF Intensity $\rightarrow$ Linear regression

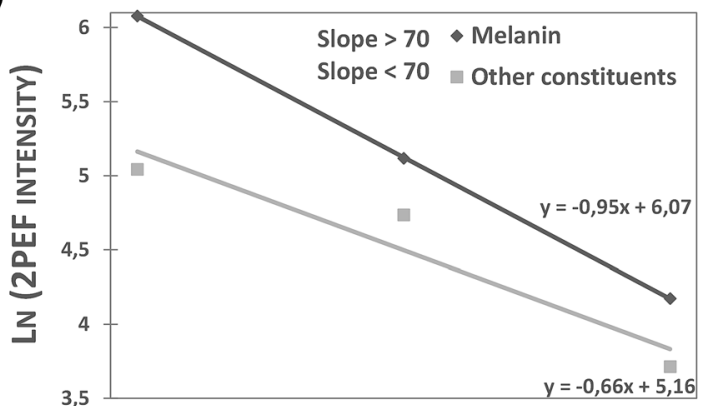

$\left.c_{2}\right)$

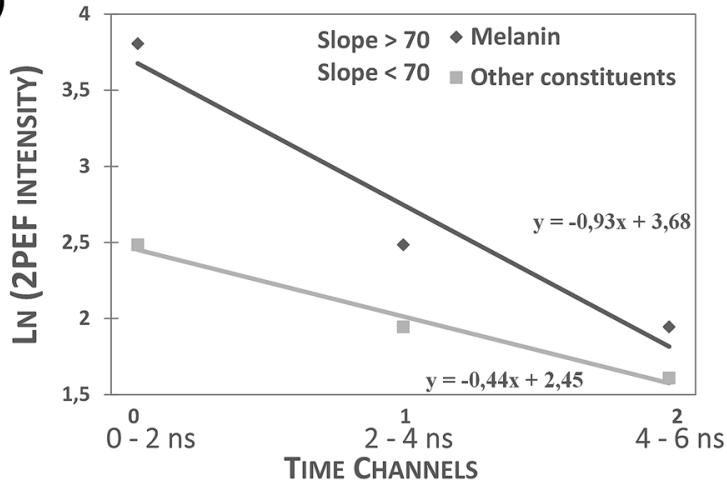

5] Melanin masks Slope $>70$ \& Pixel clusters $>1 \mu m^{2}$
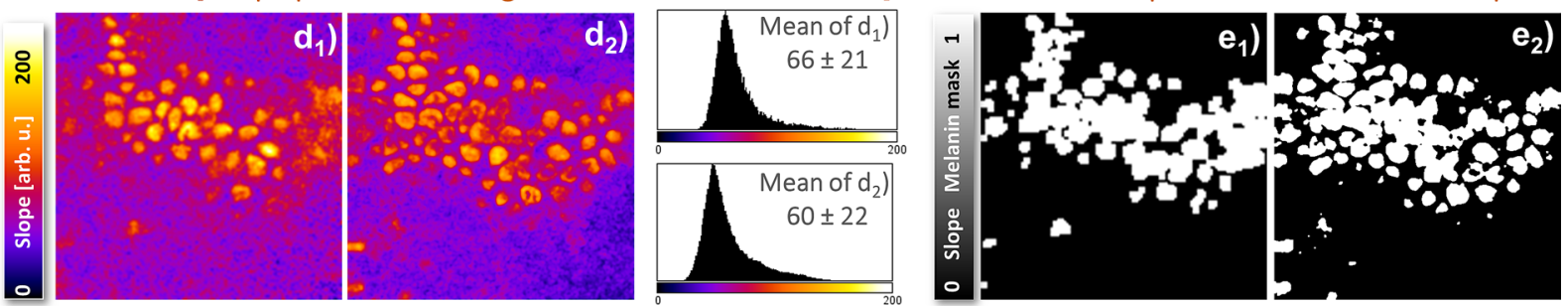

Figure 5. Effect of image acquisition time on in vivo human skin Pseudo-FLIM melanin detection. (top) 2PEF intensity images within 0-8 ns time range and within the first 4 temporally binned time channels with 2 ns integration time. (a1) 2D 2PEF-FLIM image of $127 \times 127$ pixels $\times 256$ time channels acquired within the basal layer of the epidermis (104 $\mu$ s pixel dwell time); (a2) 2D 2PEF-FLIM image of $511 \times 511$ pixels $\times 4$ time channels extracted from a $\mathrm{z}$-stack and acquired almost within the same $2 \mathrm{D}$ plane at $28 \mu$ sixel dwell time. (b1, b2) shows the corresponding $2 \mathrm{PEF}$ intensity decays of the first 3 temporally binned channels for a high slope (red arrow, melanin) and small slope (yellow arrow, other constituents) pixels. The full decay of these two pixels extracted from the $256 \mathrm{t}$-channels images is given in the insert in graph (b1). Graphs (c1, c2) show the natural logarithm transformation and linear regression fitting to extract the slopes of the decays. Images $(\mathbf{d 1}, \mathbf{d} 2)$ show the corresponding slope parametric images and their Pseudo-FLIM melanin mask is given in images $(\mathbf{e} 1, \mathbf{e} 2)$. 

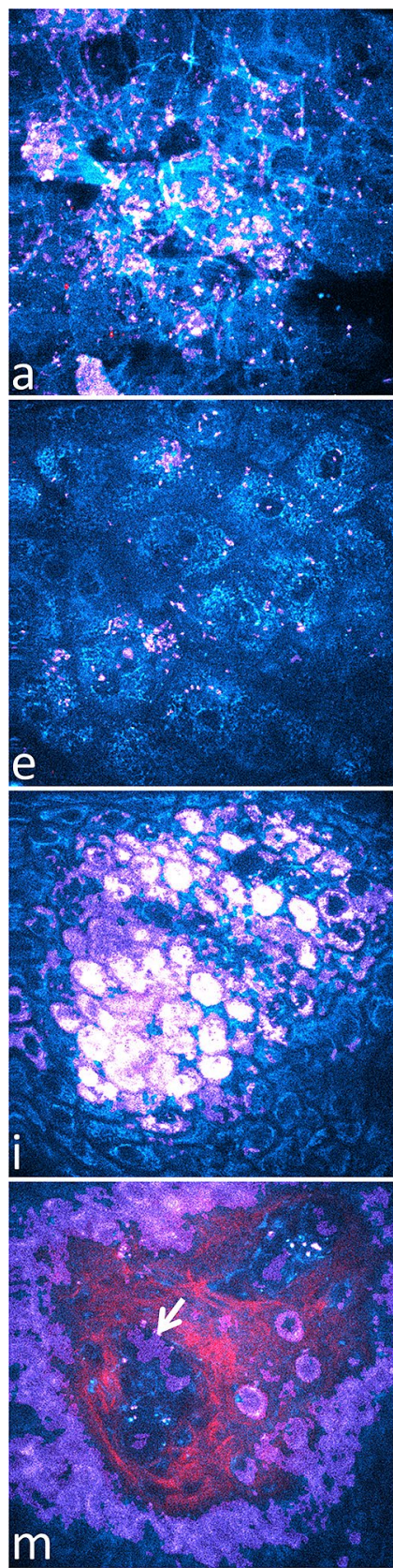
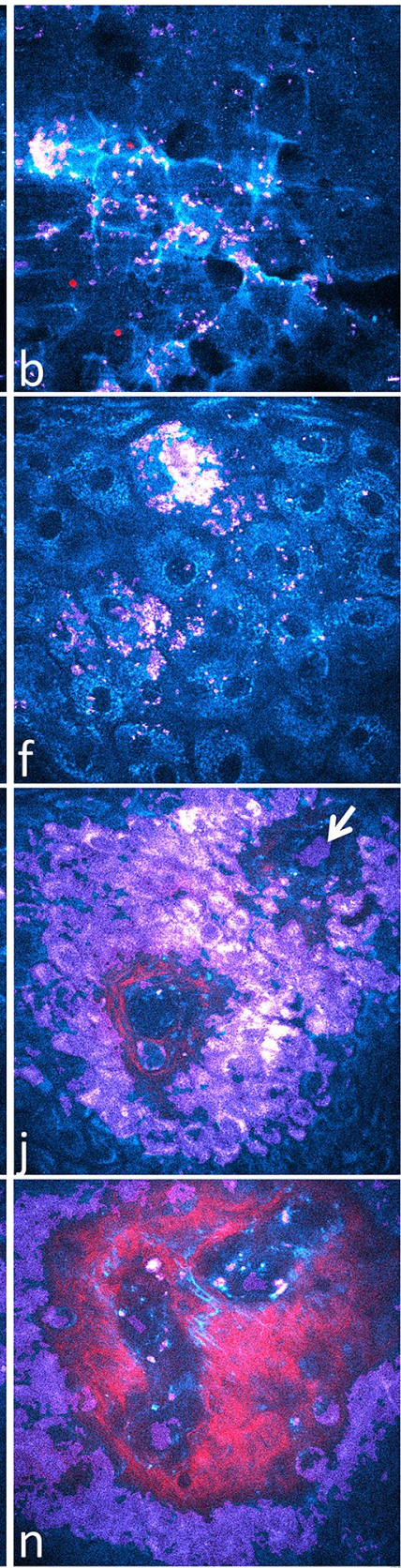
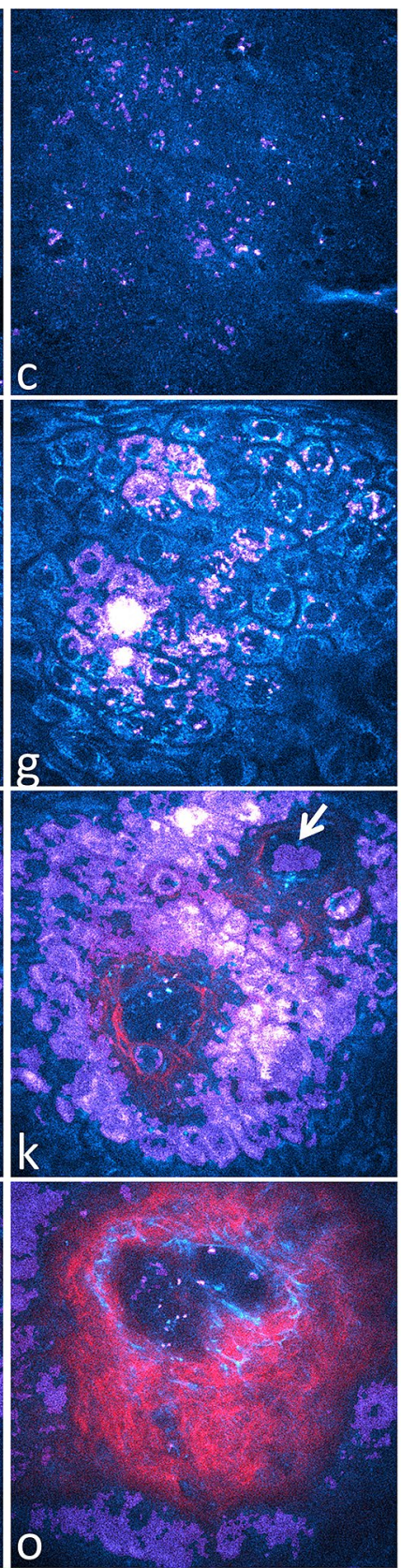
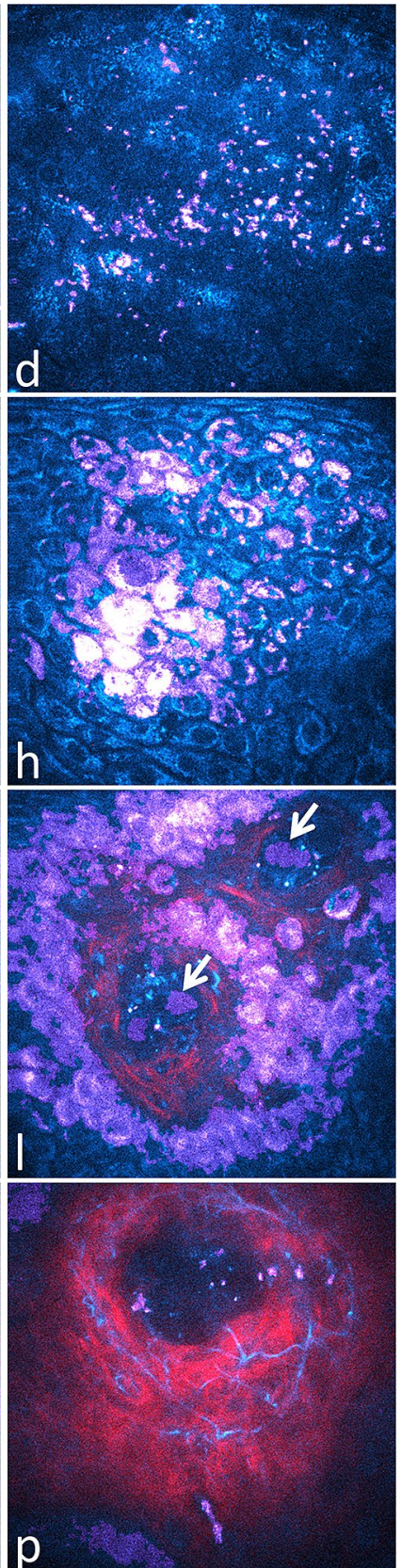

Figure 6. In vivo 3D multiphoton images of human skin - acquisition of combined 2PEF-FLIM (4 time channels)/SHG z-stacks compatible with Pseudo-FLIM melanin detection. 2PEF intensity is shown in cyan hot color, SHG in red and Pseudo-FLIM melanin mask pixels in purple. High 2PEF signal intensities appear in white color. Images are extracted from a z-stack of 70 en face images acquired with $2.346 \mu \mathrm{m}$ z-step. (a) stratum corneum disjunctum; (b) stratum corneum disjunctum/compactum interface; (c, d) compactum / granulosum interface; (e, f) stratum granulosum; $(\mathbf{g}, \mathbf{h})$ stratum spinosum; $(\mathbf{i})$ stratum basale; $(\mathbf{j}-\mathbf{o})$ stratum basale/dermis interface; (p) superficial dermis. Within the blood capillaries, Pseudo-FLIM detects cells with high slope values, fast decays, that most probably emit $2 \mathrm{PEF}$ signals from hemoglobin (see arrows). As the image acquisition time is slower than the blood flow, some blood cells appear with a deformed shape as indicated by the arrow in image $\mathrm{m}$.

on skin samples with variable constitutive pigmentation ${ }^{9}$. Although the correlations are strong, the link between skin color and melanin content is not necessarily linear: indeed, in group III, for the same measured color, the global melanin density is clearly higher in the subgroup of Asian origin (Fig. 7c). Such difference is not observed between Eu.O and As.O subjects in group II, possibly due to the very low density of melanin in light skin-type groups. Clear differences in global melanin density between Asian and European volunteers in groups III and IV (Fig. 7c) are associated with small ones for the $L^{*}, b^{\star}$ and ITA parameters (Fig. S5). The Asian volunteers have a slightly darker skin color and an increased $b^{*}$ value representing the blue-yellow component. 

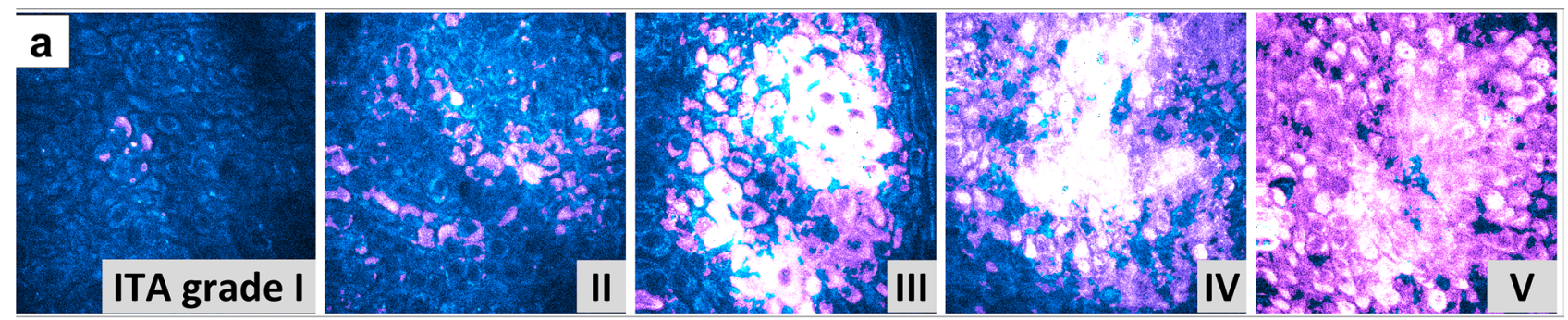

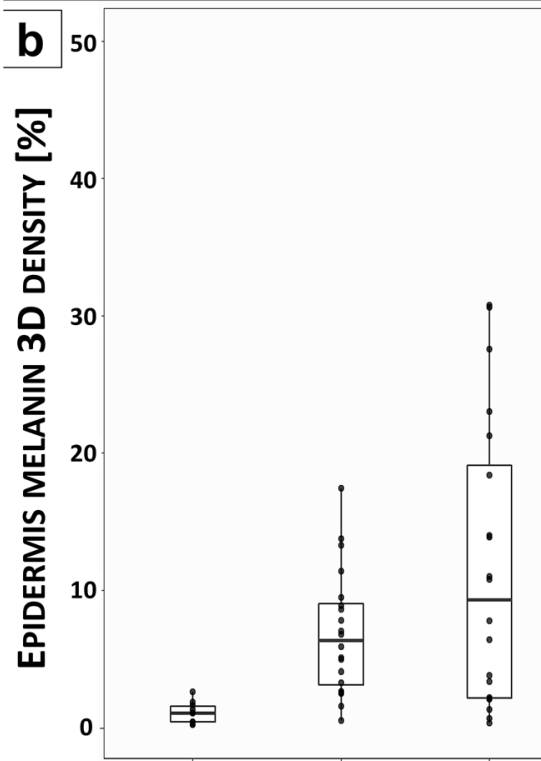

d
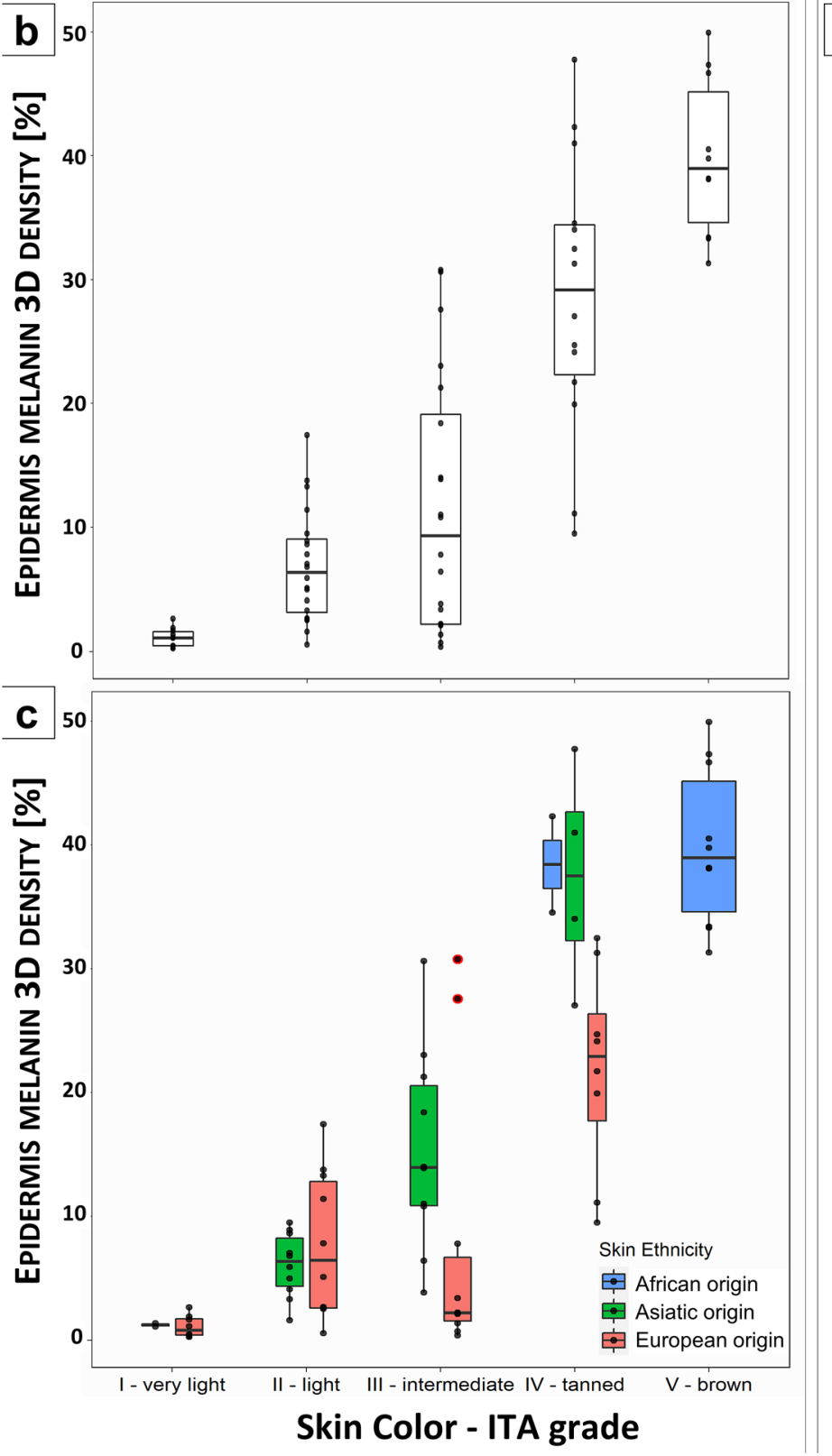

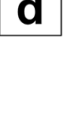

롱

CORRELATION MATRIX

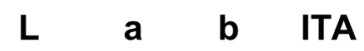

$\mathbf{L}$

$-0.89$

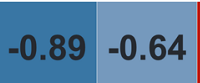

b

ITA

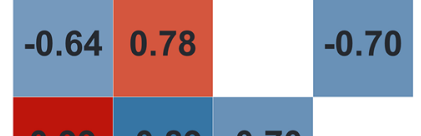

$\frac{1}{9}$

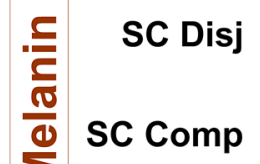

$\begin{array}{llll}-0.41 & 0.31 & 0.09 & -0.40\end{array}$

$\begin{array}{llll}-0.46 & 0.48 & 0.43 & -0.45\end{array}$

I LED

동

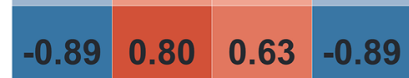

SGranu

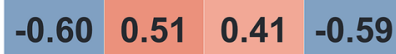

SSpin

SBas

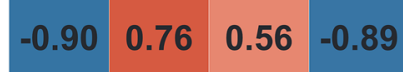

$\begin{array}{llll}-0.86 & 0.82 & 0.67 & -0.85\end{array}$

pearson

Figure 7. Modulation of global epidermal 3D melanin density with skin color ITA grade in constitutive pigmentation study. (a) Raw 2PEF intensity images (cyan hot) and Pseudo-FLIM melanin masks (purple) acquired within the basal layer of human forearm skin. High signal intensities appear in white color. (b)

Modulation of 3D epidermal melanin density with ITA group and (c) with both ITA group and skin ethnicity. The data are expressed as boxplots with fences. (d) Correlation matrix of the skin colorimetric parameters and multiphoton melanin density estimated in the epidermis and its sub-layers. The data correspond to the Pearson correlation coefficients. An absolute value of the Pearson correlation coefficients between $[0.6,0.8]$ and $[0.8,1.0]$ indicate a strong and respectively a very strong correlation. ED (global epidermis), SC - stratum corneum, SC Disj - stratum corneum disjunctum, SC Comp - stratum corneum compactum, LED - living epidermis, SGranu stratum granulosum, SSpin - stratum spinosum and SBas - stratum basale. 

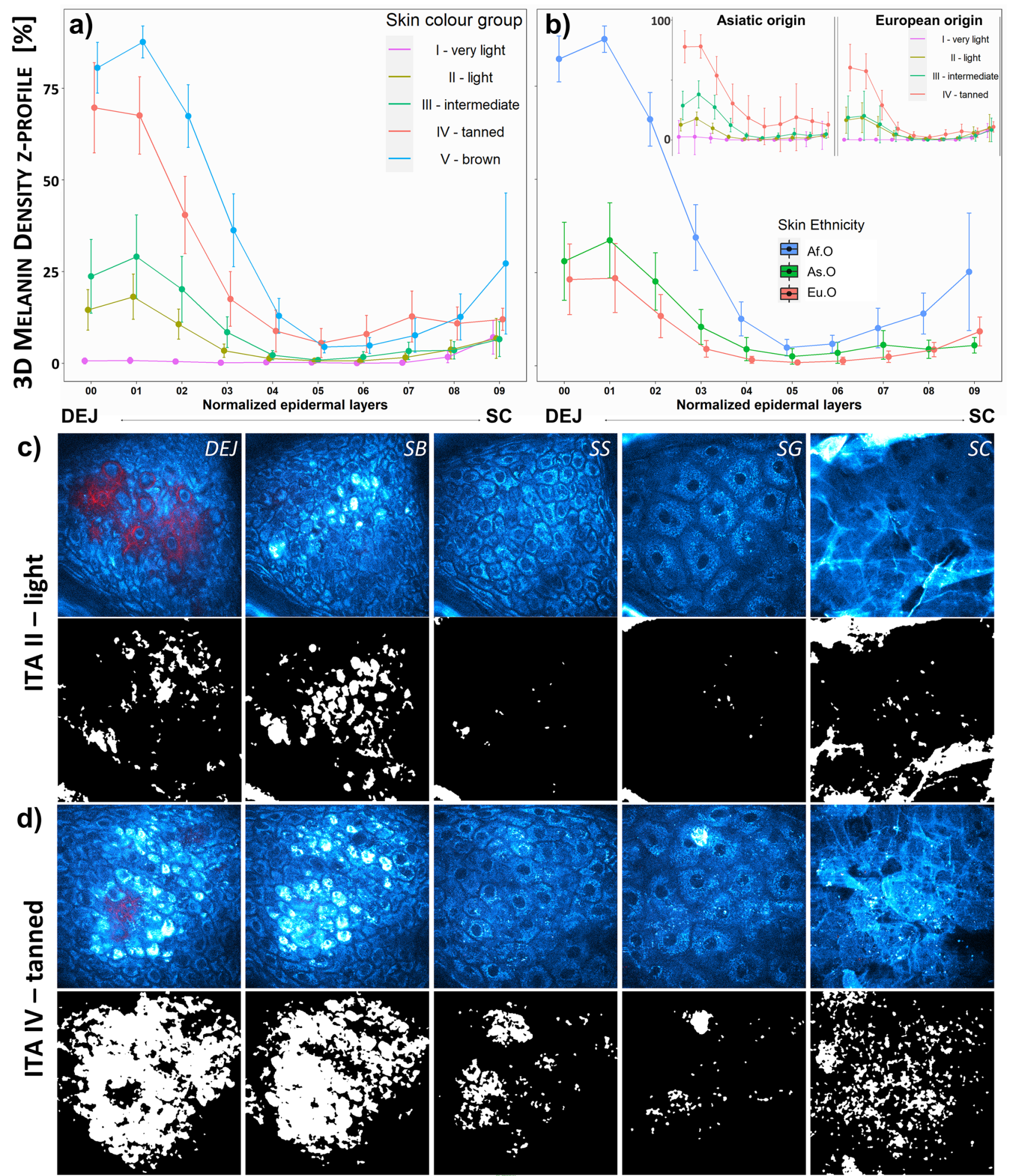

Figure 8. Modulation of $\mathrm{z}$-epidermal distribution with skin color ITA grade and skin ethnicity in constitutive pigmentation study. Melanin density z-epidermal distribution profiles (mean 3D melanin density estimated in 12 thickness-normalized epidermal layers from 1-DEJ level to 12-SC level) of (a) different skin color ITA groups and b) different skin ethnicity. The insert in (b) is showing the z-profiles of different skin color ITA groups for Asiatic and European origins. The z-profiles data are expressed as mean \pm confidence intervals of the mean. Raw 2PEF intensity images (cyan hot) and Pseudo-FLIM melanin masks (white) extracted from multiphoton z-stacks acquired on ventral forearms of volunteers with (c) light and (d) tanned skin color ITA groups. DEJ - dermal-epidermal junction level, SB - stratum basale, SS - stratum spinosum, SG - stratum granulosum and SC - stratum corneum. 
Although partly explainable by these different contributions of colorimetric parameters probably due to a different melanin composition, the significant differences in global melanin density between Asians and Europeans for a same ITA group suggest that skin color also depends on other factors as the distribution of the pigment in the thickness of the layer (see below) or on the morphology and organization of melanosomes.

Melanin density z-distribution profiles with skin color ITA group (Fig. 8a) and skin ethnicity (Fig. 8b) allow evidencing a progressive increase in melanin density at the basal and supra-basal layers for ITA grades II to V and a slight increase at the level of stratum granulosum and stratum corneum in tanned and brown skin. An example of images showing the melanin density z-differences between light and tanned skin is given in Fig. 8c, d. In group III, the epidermal melanin distribution is clearly different between Eu.O and As.O subgroups (see insert in Fig. 8b), with a significant melanin amount above the basal layer in the latter, lower however than observed in groups IV and V in which significant amounts of melanin are observed up to half of the epidermis, as well as an increase within SC (Fig. 8a). Mean epidermal thickness being comparable among ethnicities (Eu.O $60.5 \pm 9.8 \mu \mathrm{m}$, As. $65.1 \pm 10.9 \mu \mathrm{m}$ and Af.O $66.6 \pm 5.4 \mu \mathrm{m})$, this confirms in vivo that constitutive pigmentation is not only given by melanin amount, but also by its epidermal distribution.

Higher melanin density but more distributed in Asians may also explain the better UV protection of Asian skins, even light ones, compared to European skins.

Overall, the small amount of melanin detected within SC, including in the darkest skins, shows that it has little contribution to the global density, confirming that the fast decay pixels detected in some regions within the uppermost cells of SC, whether they correspond to "more keratinized" structures or to degraded melanin, have little impact on the global epidermal melanin density parameter (Fig. S6).

Chronic and seasonal sun exposure (original and published ${ }^{52}$ data). The data for chronic exposure (Fig. S7) are original data from photo-aging study comparing ventral and dorsal (mostly unexposed vs. exposed) forearms. The data for seasonal sun exposure (Fig. S9) are from retinoids 1 year-study and correspond to acquisitions performed at months M00 (March), M03 (June), M06 (September), M12 (March +1 yr) on the control untreated forearm of volunteers; these have already been published ${ }^{52}$ ).

Melanin density is obviously higher on chronically exposed zones (dorsal versus ventral forearm both in young and aged subjects, without differences between groups for a same site) (Fig. S7) and it increases progressively with summer in the 1 year-study (Fig. S9a).

Analysis of melanin z-distribution in photo-aging study shows an increased melanin density in basal and supra-basal layers in aged versus young subjects (Fig. S7f).

Among seasonality (1 year-study, control condition), the gradual increase of global density is not only due to an increase in the basal layers, but also up to the middle epidermis at month M03, and throughout the epidermis at month M06 after summer (Fig. S9c). After 1 year, a persistent accumulation of melanin in supra-basal layers, as well as at the SC level is observed when compared to M00. Thus, the progressive accumulation of melanin in the supra-basal layers of the epidermis could be part of the pigmentary changes that occur during aging and the melanin distribution profile could be a new pertinent descriptor of photoaging at least in Europeans.

Treatment (published $\mathrm{in}^{51,52}$ ). In the study of retinoids effects under occlusion ${ }^{51}$, a clear decrease of melanin content in the living epidermis is evidenced on retinol-treated area (Fig. S8), whereas no significant modification is observed at the global level after one year of usual application conditions ${ }^{52}$ (Fig. S9a). In this latter case, in retinol-treated group (Fig. S9c), although the strongest modulation of melanin distribution induced by summer (M06) isn't different compared to control group, the ascent of melanin in supra-basal layers, appearing in the control group, isn't observed at months M03 nor at M12. The melanin z-distribution at these times overlaps M00 curve for retinol-condition, strongly suggesting that in vivo, the predominant effect of retinoids on pigmentation is more likely due to skin renewal than to a direct effect on melanogenesis.

\section{Discussion and conclusions}

In this work, we implemented a 3D melanin detection approach called Pseudo-FLIM, based on slope analysis upon linear regression of temporally binned two-photon excited autofluorescence lifetime data ${ }^{48,49}$. We demonstrated by comparison with FLIM bi-exponential and phasor analyses that the fast decay shapes of melanin containing samples correspond to high slope values, a characteristic one can use to identify melanin containing pixels in vitro or in vivo. To our knowledge, this is the first time that phasor approach was applied to melanin analysis in vivo on human skin and that FLIM bi-exponential and phasor methods were compared for melanin detection. The long image acquisition time needed for acquiring enough photons per pixel for bi-exponential or phasor analysis limits the application of these methods to selected 2D epidermal or dermal depths and, in practice, is not compatible with 3D skin imaging in a clinical trial. Using Pseudo-FLIM approach, one can perform a 3D melanin detection from an entire $\mathrm{z}$-stack of in vivo multiphoton images of human skin as well as other types of melanized samples. In association with an automatic 3D epidermis segmentation ${ }^{50}$, a global 3D epidermal melanin density can be estimated. Additionally, as the epidermal thickness may vary according to the anatomical sites, skin ethnicity, aging or products application, we introduced a more refined segmentation of the epidermis that allows extracting a melanin z-epidermal distribution profile and comparing skin regions with varying epidermal thickness.

In vivo multiphoton $3 \mathrm{D}$ imaging in human volunteers has limitations in terms of image field of view $\left(\sim 130 \times 130 \mu \mathrm{m}^{2}\right.$ for DermaInspect), but the investigated volume is higher compared to histology $2 \mathrm{D} F M$ analysis ${ }^{9,10,11}$ (usually a few images of $\sim 200-250 \mu \mathrm{m}$ epidermal length are analyzed within a skin biopsy) and 2 times higher field of views can be achieved with systems such as MPTflex. Despite the small investigated epidermal volume, we have demonstrated that multiphoton global 3D epidermal melanin density is highly correlated 
with skin ITA value in constitutive pigmentation study, in agreement with ex vivo histology and HPLC chemical analysis results on skin samples with variable constitutive pigmentation ${ }^{9}$. Moreover, our results indicate that multiphoton imaging can provide quantitative data of interest in studying pigmentation modulations under different conditions (constitutive and acquired pigmentation, aging, natural UV exposure ${ }^{52}$ or application of topical retinoids ${ }^{51,52}$ or corticosteroids ${ }^{45}$ ), but its ability to accurately assess melanin heterogeneity in skin pigmentary disorders remains to be studied.

Multiphoton Pseudo-FLIM can address a wide range of in vivo situations and melanin's epidermal distribution provides new and valuable information that complements melanin content; for example, it can probably explain the difference in perceived color and photosensitivity between Asian and European skins though very similar colorimetric values. It also makes it possible to study treatments' mechanism of action, as illustrated with retinoids, whose action on melanin is due to epidermal renewal and not to a direct effect on melanin as discussed in the literature ${ }^{52}$. In another case, the progressive accumulation of melanin in the supra-basal layers of the epidermis observed during aging probably explains in part the pigmentary changes that occur over time; the demonstration of its reversion under retinoids, the gold standard of anti-aging, validates this parameter as a relevant descriptor of photoaging in Europeans.

This method could also bring new insights into the knowledge of some underlying biological mechanisms of pigmentation modulations appearing through either redistribution of existing melanin and/or de novo melanin synthesis. A still challenging topic, especially in photoprotection, is the in vivo characterization of melanin modulations upon UV exposure and their link to the clinical manifestations of hyperpigmentations, i. e. Immediate Pigment Darkening (IPD) and Persistent Pigment Darkening (PPD). Assessing melanin's global density and $3 \mathrm{D}$ distribution in vivo with multiphoton Pseudo-FLIM will avoid performing invasive biopsies and will certainly help understating the mechanisms of skin photobiology and how melanin is modulated with different UV wavelengths and doses. Knowing the importance of epidermal melanin distribution for its DNA protection factor ${ }^{69-71}$, we are confident that multiphoton Pseudo-FLIM approach will contribute to developing tomorrow's photoprotection products. More generally, the in vitro and in vivo applications of multiphoton Pseudo-FLIM based melanin detection and quantification of its global 3D density and z-epidermal distribution encompass physiological, pathological, or environmental factors-induced pigmentation modulations up to whitening, antiphotoaging, or photoprotection products evaluation.

Received: 20 August 2021; Accepted: 26 November 2021

Published online: 31 January 2022

\section{References}

1. Alaluf, S. et al. The impact of epidermal melanin on objective measurements of human skin colour. Pigment Cell Res. 15, 119-126. https://doi.org/10.1034/j.1600-0749.2002.10072.x (2002).

2. Ito, S. \& Wakamatsu, K. Quantitative analysis of eumelanin and pheomelanin in humans, mice, and other animals: A comparative review. Pigment Cell Res. 16, 523-531. https://doi.org/10.1034/j.1600-0749.2003.00072.x (2003).

3. Simon, J. D., Peles, D., Wakamatsu, K. \& Ito, S. Current challenges in understanding melanogenesis: Bridging chemistry, biological control, morphology, and function. Pigment Cell Melanoma Res. 22, 563-579. https://doi.org/10.1111/j.1755-148X.2009.00610.x (2009).

4. d'Ischia, M. et al. Melanins and melanogenesis: Methods, standards, protocols. Pigment Cell Melanoma Res. 26, 616-633. https:// doi.org/10.1111/pcmr.12121 (2013).

5. d'Ischia, M. et al. Melanins and melanogenesis: From pigment cells to human health and technological applications. Pigment Cell Melanoma Res. 28, 520-544. https://doi.org/10.1111/pcmr.12393 (2015).

6. Ito, S. \& Wakamatsu, K. Human hair melanins: What we have learned and have not learned from mouse coat color pigmentation. Pigment Cell Melanoma Res. 24, 63-74. https://doi.org/10.1111/j.1755-148X.2010.00755.x (2011).

7. Wakamatsu, K. \& Ito, S. Advanced chemical methods in melanin determination. Pigment Cell Res. 15, 174-183. https://doi.org/ 10.1034/j.1600-0749.2002.02017.x (2002).

8. Ito, S. et al. Usefulness of alkaline hydrogen peroxide oxidation to analyze eumelanin and pheomelanin in various tissue samples: Application to chemical analysis of human hair melanins. Pigment Cell Melanoma Res. 24, 605-613. https://doi.org/10.1111/j. 1755-148X.2011.00864.x (2011).

9. Del Bino, S. et al. Chemical analysis of constitutive pigmentation of human epidermis reveals constant eumelanin to pheomelanin ratio. Pigment Cell Melanoma Res. 28, 707-717. https://doi.org/10.1111/pcmr.12410 (2015).

10. Hurbain, I. et al. Melanosome distribution in keratinocytes in different skin types: Melanosome clusters are not degradative organelles. J. Invest. Dermatol. 138, 647-656. https://doi.org/10.1016/j.jid.2017.09.039 (2018).

11. Joly-Tonetti, N., Wibawa, J. I., Bell, M. \& Tobin, D. Melanin fate in the human epidermis: A reassessment of how best to detect and analyse histologically. Exp. Dermatol. 25, 501-504. https://doi.org/10.1111/exd.13016 (2016).

12. Meredith, P. \& Sarna, T. The physical and chemical properties of eumelanin. Pigment Cell Res. 19, 572-594. https://doi.org/10. $1111 / j .1600-0749.2006 .00345 . x(2006)$.

13. Watt, A., Bothma, J. \& Meredith, P. The supramolecular structure of melanin. Soft Matter https://doi.org/10.1039/B902507C (2009).

14. Teuchner, K. et al. Femtosecond two-photon excited fluorescence of melanin. Photochem. Photobiol. 70, 146-151. https://doi.org/ 10.1111/j.1751-1097.1999.tb07982.x (1999).

15. Teuchner, K. et al. Fluorescence studies of melanin by stepwise two-photon femtosecond laser excitation. J. Fluoresc. 10, $275-281$. https://doi.org/10.1023/A:1009453228102 (2000).

16. Wilhelm, K.-P., Elsner, P., Berardesca, E. \& Maibach, H. I. Bioengineering of the Skin: Skin Imaging and Analysis. 2nd edn, https:// doi.org/10.3109/9781420005516 (Informa Healthcare USA, Inc., 2007).

17. Rajadhyaksha, M., Gonzalez, S., Zavislan, J. M., Anderson, R. R. \& Webb, R. H. In vivo confocal scanning laser microscopy of human skin II: Advances in instrumentation and comparison with histology. J. Invest. Dermatol. 113, 293-303. https://doi.org/ 10.1046/j.1523-1747.1999.00690.x (1999).

18. Corcuff, P., Chaussepied, C., Madry, G. \& Hadjur, C. Skin optics revisited by in vivo confocal microscopy: melanin and sun exposure. J. Cosmet. Sci. 52, 91-102 (2001).

19. Masters, B. \& So, P. Confocal microscopy and multi-photon excitation microscopy of human skin in vivo. Opt. Express 8, 2-10. https://doi.org/10.1364/oe.8.000002 (2001). 
20. Wang, H. et al. In vivo coherent Raman imaging of the melanomagenesis-associated pigment pheomelanin. Sci. Rep. 6, 37986. https://doi.org/10.1038/srep37986 (2016).

21. Galvan, I., Araujo-Andrade, C., Marro, M., Loza-Alvarez, P. \& Wakamatsu, K. Raman spectroscopy quantification of eumelanin subunits in natural unaltered pigments. Pigment Cell Melanoma Res. 31, 673-682. https://doi.org/10.1111/pcmr.12707 (2018).

22. Huang, Z. et al. Cutaneous melanin exhibiting fluorescence emission under near-infrared light excitation. J. Biomed. Opt. 11, 34010. https://doi.org/10.1117/1.2204007 (2006).

23. Kalia, S. et al. Melanin quantification by in vitro and in vivo analysis of near-infrared fluorescence. Pigment Cell Melanoma Res. 31, 31-38. https://doi.org/10.1111/pcmr.12624 (2018).

24. Yakimov, B. P. et al. Melanin distribution from the dermal-epidermal junction to the stratum corneum: Non-invasive in vivo assessment by fluorescence and Raman microspectroscopy. Sci. Rep. 10, 14374. https://doi.org/10.1038/s41598-020-71220-6 (2020).

25. Matthews, T. E. et al. In vivo and ex vivo epi-mode pump-probe imaging of melanin and microvasculature. Biomed. Opt. Express 2, 1576-1583. https://doi.org/10.1364/BOE.2.001576 (2011).

26. Simpson, M. J. et al. Nonlinear microscopy of eumelanin and pheomelanin with subcellular resolution. J. Invest. Dermatol. 133, 1822-1826. https://doi.org/10.1038/jid.2013.37 (2013).

27. Wilson, J. W. et al. Comparing in vivo pump-probe and multiphoton fluorescence microscopy of melanoma and pigmented lesions. J. Biomed. Opt. 20, 051012. https://doi.org/10.1117/1.JBO.20.5.051012 (2015).

28. Sun, C. K. et al. Slide-free clinical imaging of melanin with absolute quantities using label-free third-harmonic-generation enhancement-ratio microscopy. Biomed. Opt. Express 11, 3009-3024. https://doi.org/10.1364/BOE.391451 (2020).

29. Zimmerley, M., Mahou, P., Débarre, D., Schanne-Klein, M.-C. \& Beaurepaire, E. Probing ordered lipid assemblies with polarized third-harmonic-generation microscopy. Phys. Rev. X 3, 011002. https://doi.org/10.1103/PhysRevX.3.011002 (2013).

30. Fellner, M. J., Chen, A. S., Mont, M., McCabe, J. \& Baden, M. Patterns and intensity of autofluorescence and its relation to melanin in human epidermis and hair. Int. J. Dermatol. 18, 722-730. https://doi.org/10.1111/j.1365-4362.1979.tb05009.x (1979).

31. Masters, B. R. \& So, P. T. Multi-photon excitation microscopy and confocal microscopy imaging of in vivo human skin: A comparison. Microsc. Microanal. 5, 282-289. https://doi.org/10.1017/s1431927699990311 (1999).

32. Pena, A.-M. et al. Multiphoton FLIM in cosmetic clinical research in Multiphoton Microscopy and Fluorescence Lifetime Imaging. Applications in Biology and Medecine (ed Karsten König) Ch. 19, 369-393 https://doi.org/10.1515/9783110429985 (De Gruyter, 2018).

33. König, K. Multiphoton microscopy and fluorescence lifetime imaging. Appl. Biol. Med. https://doi.org/10.1515/9783110429 985(DeGruyter (2018).

34. Zipfel, W. R., Williams, R. M. \& Webb, W. W. Nonlinear magic: Multiphoton microscopy in the biosciences. Nat. Biotechnol. 21, 1369-1377. https://doi.org/10.1038/nbt899 (2003).

35. Zipfel, W. R. et al. Live tissue intrinsic emission microscopy using multiphoton-excited native fluorescence and second harmonic generation. Proc. Natl. Acad. Sci. U. S. A. 100, 7075-7080. https://doi.org/10.1073/pnas.0832308100 (2003).

36. Pena, A.-M., Strupler, M., Boulesteix, T., Godeau, G. \& Schanne-Klein, M.-C. Spectroscopic analysis of keratin endogenous signal for skin multiphoton microscopy. Opt. Express 13, 6268-6274; erratum: 6213(6217)6667, https://doi.org/10.1364/OPEX.13.006268 (2005).

37. Periasamy, A. \& Clegg, R. M. FLIM Microscopy in Biology and Medicine. 1st edn, https://doi.org/10.1201/9781420078916 (Chapman and Hall/CRC, Taylor \& Francis Group, 2009).

38. Dancik, Y., Favre, A., Loy, C. J., Zvyagin, A. V. \& Roberts, M. S. Use of multiphoton tomography and fluorescence lifetime imaging to investigate skin pigmentation in vivo. J. Biomed. Opt. 18, 26022. https://doi.org/10.1117/1.JBO.18.2.026022 (2013).

39. Ehlers, A., Riemann, I., Stark, M. \& Konig, K. Multiphoton fluorescence lifetime imaging of human hair. Microsc. Res. Tech. 70, 154-161. https://doi.org/10.1002/jemt.20395 (2007).

40. Dimitrow, E. et al. Spectral fluorescence lifetime detection and selective melanin imaging by multiphoton laser tomography for melanoma diagnosis. Exp. Dermatol. 18, 509-515. https://doi.org/10.1111/j.1600-0625.2008.00815.x (2009).

41. Sugata, K. et al. Imaging of melanin distribution using multiphoton autofluorescence decay curves. Skin Res. Technol. 16, 55-59. https://doi.org/10.1111/j.1600-0846.2009.00397.x (2010).

42. Krasieva, T. B. et al. Two-photon excited fluorescence lifetime imaging and spectroscopy of melanins in vitro and in vivo. J. Biomed. Opt. 18, 31107. https://doi.org/10.1117/1.JBO.18.3.031107 (2013).

43. Sitiwin, E. et al. Shedding light on melanins within in situ human eye melanocytes using 2-photon microscopy profiling techniques. Sci. Rep. 9, 18585. https://doi.org/10.1038/s41598-019-54871-y (2019).

44. Stringari, C. et al. Phasor approach to fluorescence lifetime microscopy distinguishes different metabolic states of germ cells in a live tissue. Proc. Natl. Acad. Sci. U. S. A. 108, 13582-13587. https://doi.org/10.1073/pnas.1108161108 (2011).

45. Ait El Madani, H. et al. In vivo multiphoton imaging of human skin assessment of topical corticosteroid-induced epidermis atrophy and depigmentation. J. Biomed. Opt. 17, 026009. https://doi.org/10.1117/1.JBO.17.2.026009 (2012).

46. Qiu, J. et al. The skin-depigmenting potential of Paeonia lactiflora root extract and paeoniflorin: In vitro evaluation using reconstructed pigmented human epidermis. Int. J. Cosmet. Sci. 38, 444-451. https://doi.org/10.1111/ics.12309/epdf (2016).

47. Saager, R. B. et al. In vivo measurements of cutaneous melanin across spatial scales: Using multiphoton microscopy and spatial frequency domain spectroscopy. J. Biomed. Opt. 20, 066005. https://doi.org/10.1117/1.JBO.20.6.066005 (2015).

48. Pena, A.-M., Baldeweck, T., Tancrede, E., Decencière, E. \& Koudoro, S. Non-invasive method for specific 3D detection, visualization and/or quantification of an endogeneous fluorophore such as melanin in a biological tissue. France patent French patent FR2982369, International publication number WO2013068943 (2011).

49. Pena, A.-M. et al. In vivo 3D quantification of melanin in human skin based on multiphoton microscopy and image processing. In 28th IFSCC Congress - International Federation of Societies of Cosmetic Chemists. Proceedings of IFSCC Congress 1129-1134 (2014).

50. Decenciere, E. et al. Automatic 3D segmentation of multiphoton images: A key step for the quantification of human skin. Skin Res. Technol. 19, 115-124. https://doi.org/10.1111/srt.12019 (2013).

51. Tancrede-Bohin, E. et al. Non-invasive short-term assessment of retinoids effects on human skin in vivo using multiphoton microscopy. J. Eur. Acad. Dermatol. Venereol. 29, 673-681. https://doi.org/10.1111/jdv.12650 (2015)

52. Tancrede-Bohin, E. et al. In vivo multiphoton imaging for non-invasive time course assessment of retinoids effects on human skin. Skin Res. Technol. 26, 794-803. https://doi.org/10.1111/srt.12877 (2020).

53. Digman, M. A., Caiolfa, V. R., Zamai, M. \& Gratton, E. The phasor approach to fluorescence lifetime imaging analysis. Biophys. J. 94, L14-16. https://doi.org/10.1529/biophysj.107.120154 (2008).

54. Vallmitjana, A. et al. Resolution of 4 components in the same pixel in FLIM images using the phasor approach. Methods and Appl. Fluorescence 8, 035001. https://doi.org/10.1088/2050-6120/ab8570 (2020).

55. Lakowicz, J. R. Principles of Fluorescence Spectroscopy. 3rd edn, https://doi.org/10.1007/978-0-387-46312-4 (Springer, 2006).

56. Wasserstein, R. L. \& Lazar, N. A. The ASA statement on p-values: Context, process, and purpose. Am. Stat. 70, 129-133. https:// doi.org/10.1080/00031305.2016.1154108 (2016).

57. Lakowicz, J. R., Szmacinski, H., Nowaczyk, K. \& Johnson, M. L. Fluorescence lifetime imaging of free and protein-bound NADH. Proc. Natl. Acad. Sci. U. S. A. 89, 1271-1275. https://doi.org/10.1073/pnas.89.4.1271 (1992).

58. Bird, D. K. et al. Metabolic mapping of MCF10A human breast cells via multiphoton fluorescence lifetime imaging of the coenzyme NADH. Cancer Res. 65, 8766-8773. https://doi.org/10.1158/0008-5472.CAN-04-3922 (2005). 
59. Becker, W. Fluorescence lifetime imaging-techniques and applications. J. Microsc. 247, 119-136. https://doi.org/10.1111/j.13652818.2012.03618.x (2012).

60. Ung, T. P. L. et al. Simultaneous NAD(P)H and FAD fluorescence lifetime microscopy of long UVA-induced metabolic stress in reconstructed human skin. Sci. Rep. 11, 22171. https://doi.org/10.1038/s41598-021-00126-8 (2021).

61. Huang, S., Heikal, A. A. \& Webb, W. W. Two-photon fluorescence spectroscopy and microscopy of NAD(P)H and flavoprotein. Biophys. J. 82, 2811-2825. https://doi.org/10.1016/S0006-3495(02)75621-X (2002).

62. Balu, M. et al. In vivo multiphoton NADH fluorescence reveals depth-dependent keratinocyte metabolism in human skin. Biophys. J. 104, 258-267. https://doi.org/10.1016/j.bpj.2012.11.3809 (2013).

63. Pena, A. M. et al. Multiphoton FLIM imaging of NADH and FAD to analyze cellular metabolic activity of reconstructed human skin in response to UVA light in Multiphoton Microscopy in the Biomedical Sciences XIX 2019. (eds P. T. C. So, K. Konig, \& A. Periasamy) Progr. Biomed. Opt. Imaging Proc. SPIE 108820a https://doi.org/10.1117/12.2508858 (SPIE, 2019).

64. Balu, M. et al. Distinguishing between benign and malignant melanocytic nevi by in vivo multiphoton microscopy. Cancer Res. 74, 2688-2697. https://doi.org/10.1158/0008-5472.CAN-13-2582 (2014).

65. Zheng, W., Li, D., Zeng, Y., Luo, Y. \& Qu, J. Y. Two-photon excited hemoglobin fluorescence. Biomed. Opt. Express 2, 71-79. https:// doi.org/10.1364/BOE.2.000071 (2010).

66. Sun, Q., Zheng, W., Wang, J., Luo, Y. \& Qu, J. Y. Mechanism of two-photon excited hemoglobin fluorescence emission. J. Biomed. Opt. 20, 105014. https://doi.org/10.1117/1.JBO.20.10.105014 (2015).

67. Shirshin, E. A. et al. Two-photon autofluorescence lifetime imaging of human skin papillary dermis in vivo: Assessment of blood capillaries and structural proteins localization. Sci. Rep. 7, 1171. https://doi.org/10.1038/s41598-017-01238-w (2017).

68. Fast, A. et al. Fast, large area multiphoton exoscope (FLAME) for macroscopic imaging with microscopic resolution of human skin. Sci. Rep. 10, 18093. https://doi.org/10.1038/s41598-020-75172-9 (2020).

69. Del Bino, S., Sok, J., Bessac, E. \& Bernerd, F. Relationship between skin response to ultraviolet exposure and skin color type. Pigment Cell Res. 19, 606-614. https://doi.org/10.1111/j.1600-0749.2006.00338.x (2006).

70. Brenner, M. \& Hearing, V. J. The protective role of melanin against UV damage in human skin. Photochem. Photobiol. 84, 539-549. https://doi.org/10.1111/j.1751-1097.2007.00226.x (2008).

71. Fajuyigbe, D. et al. Melanin distribution in human epidermis affords localized protection against DNA photodamage and concurs with skin cancer incidence difference in extreme phototypes. FASEB J. 32, 3700-3706. https://doi.org/10.1096/f. 201701472R (2018).

\title{
Acknowledgements
}

We thank Hassan Ait El Madani, Marjorie Chéraud-Carpentier, Nathalie Parent, Nathalie Pineau and Steeve Victorin, for their respective contribution in carrying out the retinoids and constitutive pigmentation clinical studies.

\section{Author contributions}

A.-M.P., T.B. and E.T.-B. conceived the experiments. E.D., S.K., T.B., A.-M.P. and E.T.-B. contributed to the development of the 3D multiphoton image analysis tools. A.-M.P., E.D. S.K., T.B. and E.T.-B. are the inventors of patented Pseudo-FLIM method. P.S. prepared the NHMK coculture. A.-M.P. and S.B. acquired and analyzed the FLIM data. A.-M.P. and E.T.-B. took the lead in writing the manuscript. All authors contributed to data analysis and writing of the manuscript.

\section{Competing interests}

The authors declare that they have no competing financial interests. A-M.P., S.B., P.S., T.B. and E.T-B. are employees of L'Oréal Research and Innovation.

\section{Additional information}

Supplementary Information The online version contains supplementary material available at https://doi.org/ 10.1038/s41598-021-03114-0.

Correspondence and requests for materials should be addressed to A.-M.P.

Reprints and permissions information is available at www.nature.com/reprints.

Publisher's note Springer Nature remains neutral with regard to jurisdictional claims in published maps and institutional affiliations.

\begin{abstract}
Open Access This article is licensed under a Creative Commons Attribution 4.0 International cc) License, which permits use, sharing, adaptation, distribution and reproduction in any medium or
format, as long as you give appropriate credit to the original author(s) and the source, provide a link to the Creative Commons licence, and indicate if changes were made. The images or other third party material in this article are included in the article's Creative Commons licence, unless indicated otherwise in a credit line to the material. If material is not included in the article's Creative Commons licence and your intended use is not permitted by statutory regulation or exceeds the permitted use, you will need to obtain permission directly from the copyright holder. To view a copy of this licence, visit http://creativecommons.org/licenses/by/4.0/.
\end{abstract}

(C) The Author(s) 2022 